\title{
Comparative genomics and transcriptional profiles of Saccharopolyspora erythraea NRRL 2338 and a classically improved erythromycin over-producing strain
}

Clelia Peano ${ }^{1}$, Adelfia Talà2, Giorgio Corti ${ }^{1}$, Daniela Pasanisi ${ }^{2}$, Miriana Durante $^{3}$, Giovanni Mita ${ }^{3}$, Silvio Bicciato ${ }^{4}$, Gianluca De Bellis ${ }^{1 *}$ and Pietro Alifano ${ }^{2^{*}}$

\begin{abstract}
Background: The molecular mechanisms altered by the traditional mutation and screening approach during the improvement of antibiotic-producing microorganisms are still poorly understood although this information is essential to design rational strategies for industrial strain improvement. In this study, we applied comparative genomics to identify all genetic changes occurring during the development of an erythromycin overproducer obtained using the traditional mutate-and- screen method.

Results: Compared with the parental Saccharopolyspora erythraea NRRL 2338, the genome of the overproducing strain presents 117 deletion, 78 insertion and 12 transposition sites, with 71 insertion/deletion sites mapping within coding sequences (CDSs) and generating frame-shift mutations. Single nucleotide variations are present in 144 CDSs. Overall, the genomic variations affect 227 proteins of the overproducing strain and a considerable number of mutations alter genes of key enzymes in the central carbon and nitrogen metabolism and in the biosynthesis of secondary metabolites, resulting in the redirection of common precursors toward erythromycin biosynthesis. Interestingly, several mutations inactivate genes coding for proteins that play fundamental roles in basic transcription and translation machineries including the transcription anti-termination factor NusB and the transcription elongation factor Efp. These mutations, along with those affecting genes coding for pleiotropic or pathway-specific regulators, affect global expression profile as demonstrated by a comparative analysis of the parental and overproducer expression profiles. Genomic data, finally, suggest that the mutate-and-screen process might have been accelerated by mutations in DNA repair genes.
\end{abstract}

Conclusions: This study helps to clarify the mechanisms underlying antibiotic overproduction providing valuable information about new possible molecular targets for rationale strain improvement.

Keywords: Saccharopolyspora erythraea, Secondary metabolism, Antibiotic fermentation, Strain improvement, Comparative genomics

\footnotetext{
* Correspondence: gianluca.debellis@itb.cnr.it; pietro.alifano@unisalento.it ${ }^{1}$ Institute of Biomedical Technologies, National Research Council, Via Fratelli Cervi 93, 20090 Segrate, Milano, Italy

${ }^{2}$ Dipartimento di Scienze e Tecnologie Biologiche ed Ambientali, Università del Salento, Via Monteroni, 73100 Lecce, Italy

Full list of author information is available at the end of the article
} 


\section{Background}

Actinomycetes are ecologically important microorganisms that hold a prominent position in the industry due to their ability to produce a wide range of secondary metabolites with biological activities including antibiotics, anti-tumour agents and immuno-suppressants [1]. However, these microorganisms must often be genetically improved for higher production before they can be used in an industrial setting. Historically, strain improvement has been empirically carried out by multiple rounds of random mutagenesis and screening [2]. Since the late 1970s, the availability of molecular genetics tools and information about the biosynthetic pathways and genetic control for most of secondary metabolites of commercial interest has opened the way for improving strains through engineering-based approaches $[3,4]$. More recently, these rational strain improvement strategies benefit from the support of genomic, transcriptomic, proteomic, and metabolomic technologies [5-12]. Combining classical and recombinant strain improvement with a solid fermentation development program represents the optimal synergy to design commercially successful processes.

The erythromycin fermentation has been improved by the traditional mutate-and-screen method over the past 50 years. Erythromycin biosynthesis in the mycelial actinomycete, Saccharopolyspora erythraea, has been widely studied as a model system for antibiotic production [13-16] and erythromycin and its semi-synthetic derivatives are widely used in the clinic. As such, the development of improved producers still represents a challenging and up- to-date issue. Erythromycin A is obtained through a three-stage pathway [17], i.e., i) assembly of the 14-membered macrolactone 6-deoxyerythronolide $\mathrm{B}$ (6DEB) from one propionyl-CoA and six (2S)-methylmalonyl-CoA units by multifunctional modular polyketide synthase followed by ii) its hydroxylation to erythronolide $B(E B)$, formation of the deoxysugars mycarose and desosamine from glucose and their addition to EB to make erythromycin D, and then iii) C-12 hydroxylation and $\mathrm{C}-3$ " O-methylation of the latter compound to produce erythromycin A $[18,19]$.

Extensive genetic studies have provided some insight into the genes involved in erythromycin biosynthesis $[20,21]$. The erythromycin gene cluster contains 20 genes arranged in four major polycistronic units [22]. Evidence for regulatory genes has been missing for a long time hampering efforts to enhance erythromycin production other than by medium manipulation, random mutagenesis and selection. In recent times, the availability of the entire genome sequence of S. erythraea and the advent of metabolic engineering opened the possibility to deeply investigate the molecular mechanisms controlling erythromycin production [23-25]. Whole-genome approaches led, for instance, to the identification of BldD, a key developmental regulator in actinomycetes [26,27], as one of the major regulators of erythromycin synthesis [28]. At the same time, metabolic engineering evidenced that, manipulating the methylmalonyl-CoA metabolite node in S. erythraea and in Aeromicrobium erythreum, a non-filamentous erythromycin A producer $[29,30]$, i.e., increasing the flux through feeder metabolic pathways, strongly influences the erythromycin yields.

Lately, new global approaches based on "RNA polymerase and ribosome engineering" have been successful used to improve erythromycin production under laboratory conditions. It has been shown that several mutations affecting rpsL (coding for the ribosomal protein S12) result in a marked enhancement of erythromycin production, accompanied by increased transcription of bldD [31]. It has been reported also that several mutations in $r p o B$ (coding for the beta subunit of RNA polymerase) deeply change the transcriptional profile of $S$. erythraea. In particular, the expression of genes coding for key enzymes of carbon (and energy) and nitrogen central metabolism is dramatically altered affecting in turn the flux of metabolites through erythromycin feeder pathways [32]. Mutations in ribosomal protein- and RNA polymerase subunit-encoding genes can be easily selected in the presence of drugs opening the way for a new approach to strain improvement. Very recently, complete biosynthesis of erythromycin A and designed analogs has been obtained using $E$. coli as a heterologous host suggesting alternative strategies to improve erythromycin production [33].

The focus of the present study is to investigate the molecular mechanisms leading to erythromycin overproduction in a classically improved strain by using a genomic approach. To this purpose, we identified all genetic changes that occurred in $S$. erythraea NRRL 2338/Px (hereafter indicated as S. erythraea Px), an erythromycin overproducer obtained through the traditional mutate-and-screen method. Compared with parental $S$. erythraea NRRL 2338, a total of 117 deletion sites, 78 insertion sites and 12 transposition sites were found across the genome of the overproducer. Moreover, single nucleotide variations affecting a total of 144 CDSs were identified between the two genomes. All genetic changes have been carefully mapped in $S$. erythraea Px genome and genomic information has been used to elucidate the molecular mechanism underlying the overproduction of erythromycin by this strain. Genomic comparison has been supported by comparative transcriptome, an approach that has been also successful used before this study [34], and phenotypic analysis. 


\section{Results and discussion}

\section{Genomic comparison of S. Erythraea px and S. Erythraea} NRRL 2338

Phenotypic differences between $S$. erythraea Px and the reference strain NRRL 2338 are shown in Figure 1. With respect to NRRL 2338, Px exhibited slower growth and reduced sporulation in both Yeast Starch (YS) and Oatmeal Yeast (OMY) agar plates, less pigmentation in OMY agar, while the phenotypes of the two strains were more similar in Soluble Complete Medium (SCM) agar. SCM broth was used in fermentation and microarray experiments (see below).

To understand the genetic bases underlying phenotypic differences between the two strains and improved erythromycin A production in Px, whole genome of this strain was sequenced and compared with that of NRRL 2338. The genome of $\mathrm{Px}$ consists of a single circular chromosome of $8,212,111$ bp with an average $\mathrm{G}+\mathrm{C}$ content of $71.14 \%$. The size of the Px chromosome is 694 bp smaller than that of NRRL 2338. Genomic comparison revealed highly conserved gene content and gene order between these two strains (Figure 2A). The two genomes are $99.1 \%$ identical and there is no remarkable change in the chromosome structure as clearly shown in the dot-plot comparison.
Compared with NRRL 2338, a total of 117 deletion sites, 78 insertion sites and 12 transposition sites were found across the Px genome. Among them, 71 sites are single nucleotide insertions/deletions (indels), which affect coding sequences leading to frame-shift mutations, and only 11 are indels larger than $100 \mathrm{bp}$. The largest insertion is $1127 \mathrm{bp}$ near position 5,393,000 which is located between an oxidoreductase (SACE_4827) and a transcriptional regulator (SACE_4828), while the largest deletion is 1147 bp in 6,849,979-6,851,124 (corresponding to SACE_6108, an ATP-dependent helicase) which is located between a methyltransferase (SACE_6107) and a glycosyl transferase (SACE_6109)

Apart from frame-shift mutations, single nucleotide variations (SNVs) affecting a total of 144 CDSs were identified between the two chromosomes. The SNVs generate 110 missense and 10 nonsense mutations. The variations, also including 4 CDS duplications, 7 transposon/transposable element insertions and 1 transposon excision, affect a total of 227 proteins in the Px genome. Chromosome map of genetic variations distinguishing Px from NRRL 2338 is shown in Figure 2B. Mutations are homogeneously scattered along the whole chromosome without "core genome" vs. "non-core genome" preference. The mutated CDSs, the corresponding gene/locus names, the nature of variations

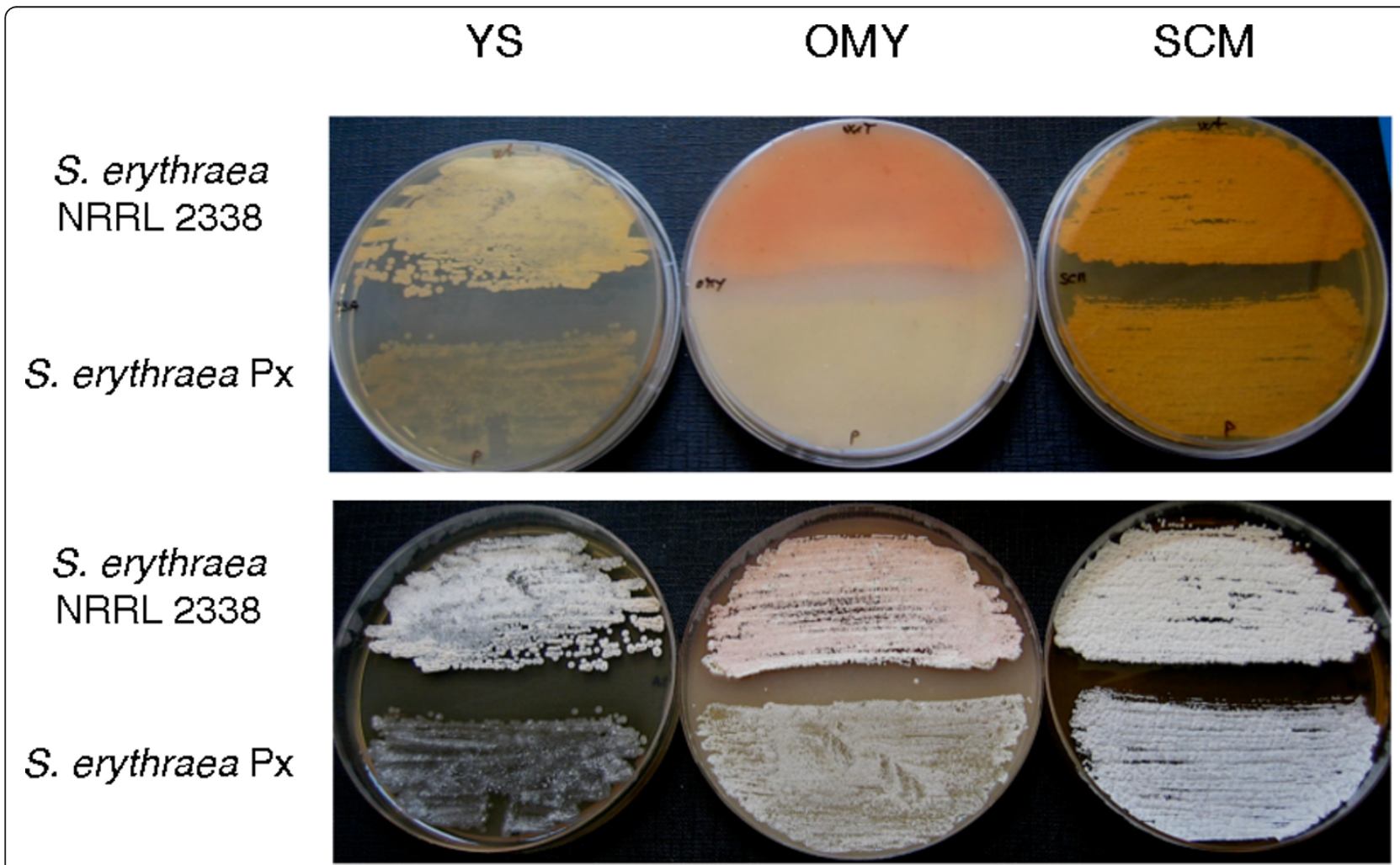

Figure 1 Phenotypes of S. erythraea NRRL 2338 and S. erythraea Px cultivated on agar media. Strains were cultivated for 7 days on YS, OMY or SCM agar plates as shown. Bottom (upper panel) and top (lower panel) sides of the agar plates were photographed. 
A

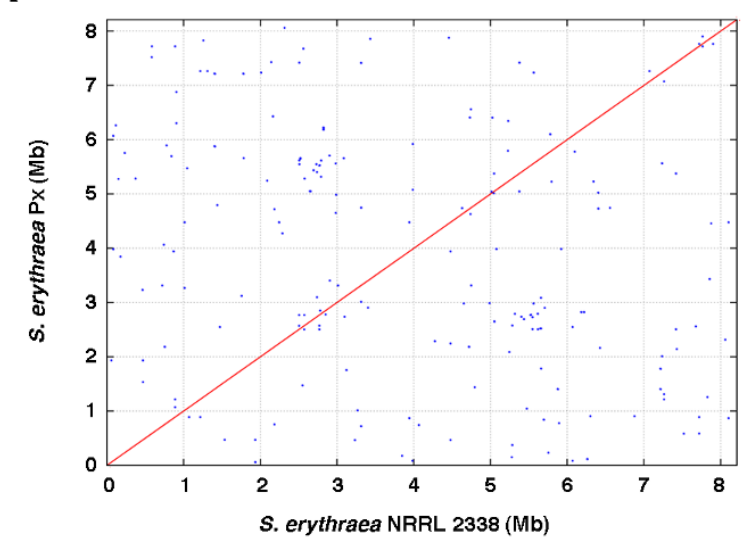

B

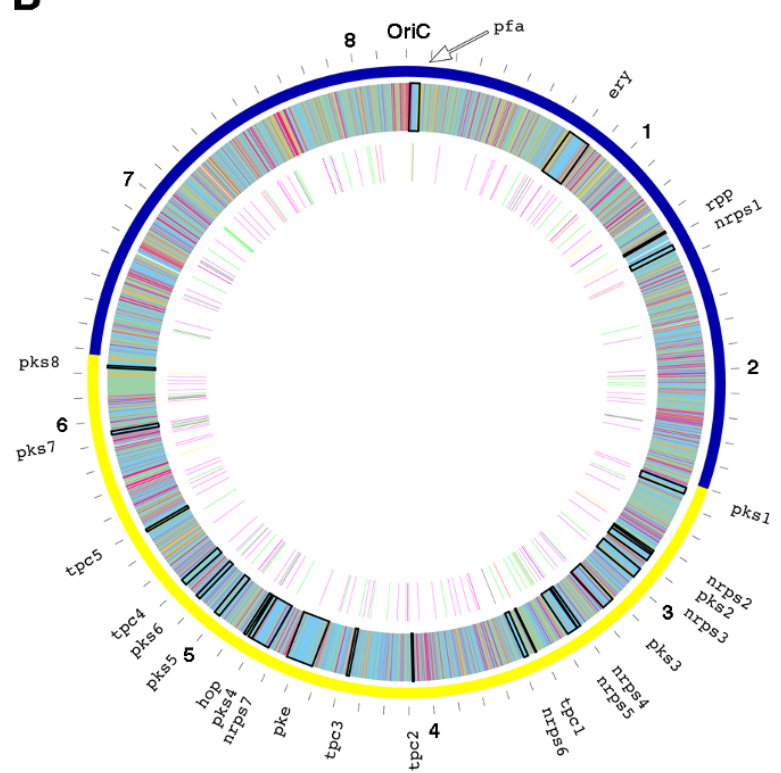

Figure 2 Dot plot and chromosome map of genetic variations distinguishing S. erythraea Px from S. erythraea NRRL 2338. A) Dot plot comparison beetwen the S. erythraea NRRL 2338 and Px strains generated by MUMmer software. B) Chromosome map of genetic variations distinguishing Px from NRRL 2338. The outer scale is numbered in megabases starting from the origin of replication (OriC), and indicates the core (blue) and noncore (yellow) regions. Outer circle: S. erythraea NRRL 2338 genescolor-coded by their COG function (orange, cellular process and signaling; purple, information storage and processing; light blue, metabolism; pale green, poorly characterized); inner circle: positions of variations between Px and NRRL 2338 color-coded by type (green, frameshift; purple, nonsense; yellow, missense). Position of secondary metabolism cluster genes are shown.

and presumed functions are reported in Tables $1,2,3,4,5$, 6 with reference to the following aspects: i.) carbon, nitrogen and sulfur metabolism; ii.) substrate uptake, membrane permeability and secretion; iii.) biosynthesis of secondary metabolites; iv.) transcription and translation processes; v.) cell division, DNA replication and repair, transposition and phage integration; vi.) CDSs of unknown functions.

\section{Transcriptome comparison of S. Erythraea px and S.} Erythraea NRRL 2338

To gain further insight about the molecular mechanisms underlying improved erythromycin A production in $\mathrm{Px}$ strain, DNA microarray of S. erythraea were manufactured and used for comparative analysis between Px and NRRL 2338. Microarray data for NRRL 2338 strains were already available [24]. In DNA microarray experiments Px and NRRL were cultivated under standard batch-culture conditions in SCM broth [29] and erythromycin production was evaluated at different time points (Figure 3).

Due to the different length of lag phase, RNA samples were collected during growth of NRRL 2338 in the time interval 12-72 $\mathrm{h}$, and in the time interval 24-84 for Px. Despite similar final values of biomass, shapes of growth 
Table 1 Variations within genes related to central carbon, nitrogen and sulfur metabolism

\begin{tabular}{|c|c|c|c|}
\hline $\begin{array}{l}\text { Gene locus in } \\
\text { NRRL } 2338\end{array}$ & $\begin{array}{l}\text { Length } \\
\text { (amino } \\
\text { acids) }\end{array}$ & $\begin{array}{l}\text { Gene/ Variation } \\
\text { locus }\end{array}$ & Protein function and notes \\
\hline
\end{tabular}

Central carbon and energy metabolism

\begin{tabular}{lllll}
\hline SACE_0618 & 286 & cCdA & Missense (G38S) & Cytochrome c biogenesis protein \\
\hline SACE_0633 & 221 & citA & IPM & Citrate synthase (N-terminal) \\
\hline SACE_1638 & 609 & suCB & Missense (V574A) & $\begin{array}{l}\text { 2-oxoglutarate dehydrogenase complex E2 component } \\
\text { (dihydrolipoamide succinyltransferase) }\end{array}$ \\
\hline SACE_3073 & 375 & hypD & Nonsense (Y30*) & Hydrogenase expression/formation protein HypD \\
\hline SACE_5291 & 335 & dhaK & Frameshift (-C 717) & Dihydroxyacetone kinase N-terminal containing protein (DhaK) \\
\hline SACE_6118 & 1125 & pyc & Frameshift (-C 1222) & Pyruvate carboxylase \\
\hline SACE_6385 & 1206 & kgd & Missense (1773 S) & 2-oxoglutarate dehydrogenase complex El component \\
\hline SACE_6668 & 288 & suCD & In frame insertion (-2081,-209V, & Succinyl-CoA synthetase, alpha subunit \\
& & & -210M, -2111,-212G, -213E) &
\end{tabular}

Nitrogen and amino acid metabolism

\begin{tabular}{lllll}
\hline SACE_0635 & 122 & UreB & Frameshift (-C 352) & Urease beta subunit \\
\hline SACE_3800 & 689 & & Missense (P601L) & $\begin{array}{l}\text { Assimilatory nitrate reductase catalytic subunit (selenocysteine- } \\
\text { Containing) }\end{array}$ \\
\hline SACE_4319 & 426 & aspB & Missense (Y185C) & Aspartate aminotransferase \\
\hline SACE_5299 & 433 & & Missense (G103R) & D-amino acid deaminase \\
\hline SACE_5427 & 417 & & Frameshift & D-amino acid dehydrogenase, small \\
\hline SACE_5741 & 500 & gltD & Frameshift (-G 1301) & Glutamate synthase NADH/NADPH, small subunit \\
\hline SACE_6330 & 421 & & Frameshift (-G 324) & Fusion protein (ligase/carboxylase and argininosuccinate lyase) \\
\hline SACE_6764 & 378 & & Missense (T47A) Frameshift (-C 436) & Alanine racemase \\
\hline Sulfur and amino acid metabolism & & \\
\hline SACE_3346 & 517 & & Missense (C286R) & Sulfite oxidase/oxidoreductase, molybdopterin binding \\
\hline SACE_4434 & 269 & tauC & Missense (L221F) & Taurine transport system permease protein TauC \\
\hline SACE_4651 & 279 & tauD & Missense (G177S) & Taurine dioxygenase, 2-oxoglutarate-dependent \\
\hline SACE_6133 & 158 & cdo2 & Missense (P151S) & Cysteine dioxygenase \\
\hline Sugar & &
\end{tabular}

\section{Sugar}

metabolism

\begin{tabular}{lllll}
\hline SACE_0652 & 383 & iroB & Missense (A325V) & Glycosyl transferase, related to UDP-glucuronosy transferase \\
\hline SACE_1940 & 788 & lacZ2 & Missense (A769V) & Beta-galactosidase/beta-glucuronidase \\
\hline SACE_3069 & 207 & gmhA & Frameshift (-G 429) & Phosphoheptose isomerase \\
\hline SACE_3071 & 223 & gmhA & $\begin{array}{l}\text { Frameshift (-C 75,-C 90, -C91, -T 95, } \\
\text {-G 98, -G 99, -C 100, -G 126) }\end{array}$ & Phosphoheptose isomerase \\
\hline SACE_3737 & 1001 & ama2 & $\begin{array}{l}\text { Missenses (R121A,N122Q, T123A, } \\
\text { F124L, I125H, V127R) }\end{array}$ & Alpha-mannosidase \\
\hline SACE_4596 & 814 & & Missense (L810Q) & Beta-galactosidase/beta-glucuronidase \\
\hline SACE_5147 & 1099 & embC & Nonsense (S399*) & Arabinosyl transferase \\
\hline SACE_5208 & 1098 & embC & Missense (S799F) & Arabinosyl transferase \\
\hline SACE_5734 & 657 & & Frameshift (+A 1027/1028) & Galactofuranosyl transferase \\
\hline SACE_6416 & 201 & $r m / C$ & Missense (A173T) & dTDP-4-dehydrorhamnose 3,5-epimerase \\
\hline SACE_6564 & 328 & deoC & Missense (G16S) & Deoxyribose-phosphate aldolase \\
\hline SACE_6765 & 501 & & Missense (R207G, P208R) & Ribokinase \\
\hline SACE_6971 & 433 & & Missense (S23F) & Glycosyl transferase \\
\hline $\begin{array}{l}\text { Fatty acid } \\
\text { metabolism }\end{array}$ & & & Missense (A211T) & Esterase \\
\hline SACE_0351 & 281 & estC & & \\
\hline
\end{tabular}


Table 1 Variations within genes related to central carbon, nitrogen and sulfur metabolism (Continued)

\begin{tabular}{|c|c|c|c|c|}
\hline SACE_3132 & 275 & $a b m C$ & $\begin{array}{l}\text { Missense (V246G) Frameshift (-C } \\
742)\end{array}$ & $\begin{array}{l}\text { Enoyl-CoA hydratase/isomerase (rev hydr) Putative: } 2 \text { - } \\
\text { ketocyclohexanecarboxyl- CoA hydrolase (amino benzoate } \\
\text { degradation) }\end{array}$ \\
\hline SACE_3216 & 526 & & Frameshift (-G 1366) & Long-chain acyl-CoA synthetase \\
\hline SACE_3361 & 329 & & Missense (T312I) & Glycerophosphoryl diester phosphodiesterase \\
\hline SACE_3450 & 304 & & IPM & Enoyl-CoA hydratase/isomerase \\
\hline SACE_3478 & 568 & & IPM & Long-chain acyl-CoA synthetase \\
\hline SACE_3745 & 255 & & Missense (L187R) & Enoyl-CoA hydratase/isomerase \\
\hline SACE_3936 & 246 & & Missense $(\mathrm{L} 48 \mathrm{H})$ & 3-oxoacyl-[acyl-carrier protein] reductase \\
\hline SACE_4589 & 388 & & Frameshift (+CACC 10/11) & Acyl-CoA dehydrogenase \\
\hline \multicolumn{5}{|l|}{$\begin{array}{l}\text { Nucleotide } \\
\text { metabolism }\end{array}$} \\
\hline SACE_1282 & 360 & $n r d B$ & Missense (S2N) & Ribonucleotide-diphosphate reductase subunit $\beta$ \\
\hline SACE_2080 & 432 & pyrC & Missense (A90V) & Dihydroorotase \\
\hline SACE_6664 & 521 & purH & Missense (P54S) & $\begin{array}{l}\text { Bifunctional phosphoribosylaminoimidazolecarboxa mide } \\
\text { formyltransferase/IMP cyclohydrolase No paralogue. }\end{array}$ \\
\hline SACE_7125 & 538 & purF & Missense (P497S) & $\begin{array}{l}\text { Glutamine phosphoribosylpyrophosphate amidotransferase No } \\
\text { paralogue }\end{array}$ \\
\hline SACE_2398 & 165 & prsA & Missense (T123P) & Phosphoribosylpyrophosphate synthetase \\
\hline SACE_5196 & 506 & $\operatorname{cod} A$ & Missense (Y322S) & Cytosine deaminase \\
\hline \multicolumn{5}{|c|}{ Vitamin and cofactor metabolism } \\
\hline SACE_0506 & 376 & thiO & Missense (A44V) & $\begin{array}{l}\text { Amino acid oxidase flavoprotein ThiO, putativeGlycine/D-amino acid } \\
\text { oxidases }\end{array}$ \\
\hline$\overline{\text { SACE_0511 }}$ & 547 & thic & Missense (T30l) & Thiamine biosynthesis protein ThiC \\
\hline SACE_5955 & 1201 & $\operatorname{cobN}$ & Missense (A1091T) & Cobaltochelatase subunit CobN \\
\hline \multicolumn{5}{|c|}{ Monooxygenases/oxidoreductases/methyltransferases/hydrolases of unknown function } \\
\hline SACE_0651 & 422 & сурА & Frameshift $(+C$ 16/17; + C 22/23) & Cytochrome P450 \\
\hline SACE_0781 & 191 & & Missense (P80S) & NADPH-dependent FMN reductase \\
\hline SACE_4325 & 341 & & Frameshift (-C 890) & Radical SAM family protein Fe-S oxidoreductase \\
\hline SACE_4560 & 326 & & Frameshift (+A 58/59) & SAM-dependent methyltransferase \\
\hline SACE_4563 & 515 & & Missense (S9F) & Coproporphyrinogen III oxidase \\
\hline SACE_4854 & 366 & & Frameshift (+G 477/478) & Amine oxidase, flavin-containing protein \\
\hline SACE_5012 & 454 & & Missense (G114E) & Haem peroxidase \\
\hline SACE_5030 & 372 & & Frameshift (-G 553) & Oye family NADH-dependent flavin oxidoreductase \\
\hline SACE_5053 & 229 & & Missense (L204F) & Amidohydrolase 2 \\
\hline SACE_6588 & 491 & & Frameshift (-C 633) & Monooxygenase, FAD-binding \\
\hline SACE_7243 & 462 & & Nonsense (W188*) & FAD-dependent oxygenase/FAD/FMN-containing dehydrogenase \\
\hline
\end{tabular}

and erythromycin production curves were markedly different between the two strains. Erythromycin production by both strains was detectable after $12 \mathrm{~h}$. Consistently with previous findings [24], three distinct phases could be distinguished during erythromycin fermentation in the reference strain: an initial period of rapid increase of antibiotic concentration lasting until $60 \mathrm{~h}$ (phase a), followed by a period of production slowdown until $72 \mathrm{~h}$ (phase $\mathbf{b}$ ), and a second period of moderate increase of erythromycin titers from 72 to $84 \mathrm{~h}$ (phase c) before entering the stationary phase. In the erythromycin overproducer phase a was protracted longer up to $72 \mathrm{~h}$, phase b lasted until $84 \mathrm{~h}$, while phase c was characterized by gradual increase of erythromycin titers until 108 $\mathrm{h}$ with a further period of production slowdown between 108 and $120 \mathrm{~h}$ followed by a further increase up to 132. Therefore, while during growth of NRRL 2338 erythromycin titers remained stable over the stationary phase, during growth of Px the antibiotic concentration continued to increase in the exhausted medium after entering the stationary phase reaching final titers about five-fold higher than in NRRL 2338. 
Table 2 Variations within genes related to substrate uptake, membrane permeability and secretion

\begin{tabular}{|c|c|c|c|c|}
\hline $\begin{array}{l}\text { Gene locus } \\
\text { in NRRL } \\
2338\end{array}$ & $\begin{array}{l}\text { Length } \\
\text { (amino } \\
\text { acids) }\end{array}$ & $\begin{array}{l}\text { Gene/ } \\
\text { locus }\end{array}$ & Variation $^{a}$ & Protein function and notes \\
\hline \multicolumn{5}{|c|}{ Transport system } \\
\hline SACE_0429 & 646 & & Missense (P416L) & $\begin{array}{l}\text { ABC-type transport system, } \\
\text { permease component }\end{array}$ \\
\hline$\overline{\text { SACE_0924 }}$ & 482 & & Frameshift (-C212;-C 221;-C 228; -G 264; -G 273) & Extracellular solute binding protein \\
\hline SACE_0925 & 337 & & $1 \mathrm{PM}$ & $\begin{array}{l}\text { ABC-type transport system, } \\
\text { permease component }\end{array}$ \\
\hline SACE_0926 & 284 & & Missense (V18M, A89T) & $\begin{array}{l}\text { ABC-type sugar transport system, } \\
\text { permease component }\end{array}$ \\
\hline$\overline{\text { SACE_0991 }}$ & 300 & & Frameshift (-G 370) & $\begin{array}{l}\text { Dipeptide transport system } \\
\text { permease }\end{array}$ \\
\hline SACE_1587 & 297 & & Frameshift (-C 585) & Sugar transport system permease \\
\hline SACE_1969 & 152 & & Missense (L90R) & $\begin{array}{l}\text { Twin-arginine translocation } \\
\text { pathway signal }\end{array}$ \\
\hline SACE_2131 & 278 & & Missense (L275F) & $\begin{array}{l}\text { Permease of the drug/metabolite } \\
\text { transporter (DMT) superfamily }\end{array}$ \\
\hline SACE_2701 & 276 & & Frameshift (-C 750) & $\begin{array}{l}\text { ABC-type transport system, ATP } \\
\text { binding component }\end{array}$ \\
\hline SACE_3038 & 545 & & Frameshift (+C 1407/1408) & $\begin{array}{l}\text { ABC-type transport system, ATP } \\
\text { binding component }\end{array}$ \\
\hline SACE_3524 & 489 & & Frameshift (+G 976/977) & $\begin{array}{l}\text { Permease of the major facilitator } \\
\text { superfamily }\end{array}$ \\
\hline SACE_4034 & 426 & & Missense (L221P) & $\begin{array}{l}\text { Permease of the major facilitator } \\
\text { superfamily }\end{array}$ \\
\hline SACE_4066 & 343 & & Frameshift (+C 268/269) & $\begin{array}{l}\text { C4 dicarboxylate transporter/malic } \\
\text { acid transport protein }\end{array}$ \\
\hline SACE_4307 & 309 & & Missense (A90T) & $\begin{array}{l}\text { Integral membrane transport } \\
\text { protein }\end{array}$ \\
\hline SACE_4347 & 555 & & Missense (A277G) & Sodium:solute symporter \\
\hline$\overline{\text { SACE_4454 }}$ & 466 & & Missense (G348S) & $\begin{array}{l}\text { Permease of the major facilitator } \\
\text { superfamily }\end{array}$ \\
\hline SACE_4982 & 407 & & Missense (L145F) & $\begin{array}{l}\text { Integral membrane transport } \\
\text { protein }\end{array}$ \\
\hline SACE_5435 & 688 & & $\begin{array}{l}\text { Missense and in frame deletion (N244T, G245A, A246P, D247D, R248A, } \\
\text { L249A, H250R, G251R, G252L, L253T, Q255C, L256A, Q258H, A259R, T260G, } \\
\text { L263-, P264-, T254S) }\end{array}$ & $\begin{array}{l}\text { ABC-type transport system, } \\
\text { permease component }\end{array}$ \\
\hline SACE_5787 & 318 & & Missense (G300R) & $\begin{array}{l}\text { Dipeptide ABC transporter, ATP- } \\
\text { binding protein }\end{array}$ \\
\hline SACE_6087 & 200 & $k d p C$ & IPM & $\begin{array}{l}\text { Potassium-transporting ATPase, } C \\
\text { chain }\end{array}$ \\
\hline$\overline{\text { SACE_6246 }}$ & 229 & & Missense (R206C) & $\begin{array}{l}\text { Phosphoenolpyruvate-protein } \\
\text { phosphotransferase }\end{array}$ \\
\hline$\overline{\text { SACE_6319 }}$ & 455 & & Frameshift (-C 1095) & $\begin{array}{l}\text { Permease of the major facilitator } \\
\text { superfamily }\end{array}$ \\
\hline$\overline{\text { SACE_6323 }}$ & 315 & & $\begin{array}{l}\text { Frameshift } \\
\text { (-AT } 913 \text { and 914) }\end{array}$ & $\begin{array}{l}\text { Dipeptide/oligopeptide/Nichel ABC } \\
\text { transporter, permease component }\end{array}$ \\
\hline$\overline{\text { SACE_6326 }}$ & 444 & & Frameshift (-G 1036) & $\begin{array}{l}\text { Permease of the major facilitator } \\
\text { superfamily }\end{array}$ \\
\hline SACE_6578 & 364 & & Missense (G270R) & $\begin{array}{l}\text { ABC-type sugar transport system, } \\
\text { permease component }\end{array}$ \\
\hline$\overline{\text { SACE_6927 }}$ & 392 & & Missense (P121L) & $\begin{array}{l}\text { Permease of the drug/metabolite } \\
\text { transporter (DMT) superfamily }\end{array}$ \\
\hline$\overline{\text { SACE_6972 }}$ & 314 & & Missense (L224F) & $\begin{array}{l}\text { Permease of the major facilitator } \\
\text { superfamily }\end{array}$ \\
\hline
\end{tabular}


Table 2 Variations within genes related to substrate uptake, membrane permeability and secretion (Continued)

\begin{tabular}{|c|c|c|c|}
\hline SACE_7047 & 403 & Frameshift (+T 654/655) & $\begin{array}{l}\text { Major facilitator superfamily sugar } \\
\text { transporter }\end{array}$ \\
\hline SACE_7202 & 327 & Frameshift (+C 873/874) & $\begin{array}{l}\text { Integral membrane transport } \\
\text { protein }\end{array}$ \\
\hline \multicolumn{4}{|c|}{ Exo-enzymes } \\
\hline$\underline{\text { SACE_1076 }}$ & ChiA2 & Nonsense (W315*) & Chitinase A \\
\hline SACE_3366 & 364 & Insertion of SACE-2828 (transposase) & Feruloyl esterase \\
\hline SACE_3961 & 296 & Missense (W21C) & $\begin{array}{l}\text { Phospholipase D/diacylglycerol } \\
\text { kinase }\end{array}$ \\
\hline SACE_6961 & 263 & Frameshift (-C 729) & $\begin{array}{l}\text { Phospholipase D/diacylglycerol } \\
\text { kinase }\end{array}$ \\
\hline \multicolumn{4}{|c|}{ Membrane and cell wall structures/enzymes } \\
\hline SACE_1186 & 202 & Frameshift (+C 84/85) & Lipoprotein \\
\hline SACE_2081 & 178 & Missense (G62D) & Integral membrane protein \\
\hline SACE_2338 & 338 & Missense (P299R) & $\begin{array}{l}\text { Membrane-bound lytic murein } \\
\text { transglycosylase B }\end{array}$ \\
\hline SACE_4420 & 192 & Frameshift (-C 79) & Lipoprotein \\
\hline SACE_5285 & 645 & Frameshift (-T 29, +C 1890/1891) & Lipoprotein \\
\hline
\end{tabular}

aPM, imperfect match

Moreover, while NRRL 2338 produced essentially erythromycin A during fermentation, Px yielded erythromycin A along with significant amounts of its immediate precursors erythromycin B and erythromycin C (Figure 3).

Gene expression data were analyzed to identify transcripts modulated during the growth curve. Considering each time point replicate as an independent entry and setting the confidence threshold at q-value $\leq 0.001$ (see Methods), the EDGE algorithm identified a total of 404 differentially expressed genes (DEGs) in NRRL 2338 (6.22\% of total probeset) with 220, 32 and 152 genes up-regulated, respectively, during phase $\mathbf{a}, \mathbf{b}$ and $\mathbf{c}$. In the Px strain the number of DEGs identified increased to 577 (8.88\% of total probeset) and only two clusters of genes up-regulated, respectively during phases a (459 genes) and phase b (104 genes) could be clearly distinguished. Microarray analysis confirmed changes in global control of cell cycle in Px strain with respect to NRRL 2338 (Figure 4).

Inspection of functional classification of cell cycleregulated genes showed that the increment in the number of DEGs in Px was mostly due to genes belonging to the following clusters of orthologous groups (COGs): I.5 Intracellular trafficking, secretion, and vescicular transport; I.6 Posttranslational modification, protein turnover, chaperones (in the functional category Cellular processes and signaling); II.12 Translation, ribosomal structure and biogenesis; III.8 Nucleotide transport and metabolism; III.9 Posttranslational modification, protein turnover, chaperones (in the functional category Metabolism) (Table 7). At the same time the following clusters of orthologous group resulted under-represented: II.11 Transcription and III.3 Cell wall/membrane/envelope biogenesis. In Table 7 the COGs have been considered over- represented (bold numbers in Table 7) if the percentage of the genes belonging to each category respect to the total of DEGs resulted 2 times more than the percentage of the genes belonging to each category respect to the total of GeneChip probesets. On the contrary they were considered under-represented (oblique numbers in Table 7) if the percentage of the genes belonging to each category respect to the total of DEGs resulted less than half of the percentage of the genes belonging to each category respect to the total probesets. A complete list of the cell cycle-regulated genes in NRRL 2338 and Px is available as supplemental data files (Additional files 1, 2), while the lists of genes up-regulated during phase a either in both strains or in Px or in NRRL 2338 are reported, respectively, in Additional files 3, 4, 5 .

The Locally Adaptive Statistical Procedure (LAP) [35] of the PREDA package [ref] was used to identify differentially expressed chromosomal regions (Figure 5). LAP analysis showed significant up-regulation of many genes clustered in the "core" region of the chromosome (mostly containing essential genes) in both NRRL 2338 and Px strains during phase a, including the erythromycin biosynthetic cluster (ery) and the ribosomal proteins-encoding operons (Figure 5A and 5B). In contrast most of genes clustering in the "non-core" region of the chromosome (mostly containing "contingency" genes) were down-regulated during this growth phase. The only notable exception was represented by $p k s 6$ cluster 
Table 3 Variations within genes related to biosynthesis of secondary metabolites

\begin{tabular}{|c|c|c|c|c|}
\hline $\begin{array}{l}\text { Gene locus in } \\
\text { NRRL } 2338\end{array}$ & $\begin{array}{l}\text { Length } \\
\text { (amino } \\
\text { acids) }\end{array}$ & $\begin{array}{l}\text { Gene } \\
\text { (locus) }\end{array}$ & Variation $^{\mathrm{a}}$ & Protein function and notes \\
\hline \multicolumn{5}{|c|}{ Polyketide synthases and related biosynthetic proteins } \\
\hline$\underline{\text { SACE_0019 }}$ & 95 & (pfa) & Nonsense (Q86*) & Acyl carrier protein \\
\hline SACE_0022 & 752 & $\begin{array}{l}\mathrm{pfaB} \\
(\mathrm{pfa})\end{array}$ & $\begin{array}{l}\text { Frameshift }(+T \\
2003 / 2004)\end{array}$ & Modular polyketide synthase (pfa gene cluster) (module 1: KR-ACP) \\
\hline SACE_0023 & 2322 & $\begin{array}{l}\mathrm{pfaC} \\
(\mathrm{pfa})\end{array}$ & $\begin{array}{l}\text { Missense (A317T, } \\
\text { D1707N) }\end{array}$ & Modular polyketide synthase (pfa gene cluster) (module 2: KS-TE) \\
\hline SACE_0718 & 237 & $\begin{array}{l}\text { eryCVI } \\
\text { (ery) }\end{array}$ & Missense (T64A) & Erythromycin biosynthesis: TDP-desosamine-N-dimethy transferase \\
\hline SACE_0720 & 322 & $\begin{array}{l}\text { eryBIV } \\
\text { (ery) }\end{array}$ & Missense (G3E) & Erythromycin biosynthesis: dTDP-4-keto-6-deoxy-L-hexose 4-reductase \\
\hline SACE_2595 & 2368 & & $\begin{array}{l}\text { Missense (G1215S, } \\
\text { D1118N) }\end{array}$ & Type I polyketide synthase (module: KS-AT-DH-ER-KR-ACP) \\
\hline SACE_2630 & 4576 & $\begin{array}{l}\text { pks2-I } \\
(p k s 2)\end{array}$ & Missense (A3004V) & $\begin{array}{l}\text { Modular polyketide synthase (module 1: KS-AT(P)-ACP; module 2: KS-AT(A)-DH-KR- } \\
\text { ACP; module 3: KS-AT(A)-DH-KR-ACP) }\end{array}$ \\
\hline SACE_2875 & 4132 & (pks3) & $\begin{array}{l}\text { Frameshift (-G } \\
\text { 2035) }\end{array}$ & $\begin{array}{l}\text { Modular polyketide synthase (pks3 gene cluster (load: CL-ACP; module 1: KS-AT(P)- } \\
\text { DH-ER-KR-ACP; module 2: KS-AT(A)-ACP) }\end{array}$ \\
\hline SACE_2876 & 252 & $\begin{array}{l}\text { gdmF } \\
\text { (pks3) }\end{array}$ & Missense (G17W) & 3-amino-5-hydroxybenzoic acid synthase (AHBA synthase) \\
\hline SACE_2888 & 455 & & Missense (V91C) & Aromatic-L-amino-acid decarboxylase \\
\hline SACE_4140 & 3481 & $\begin{array}{l}\text { pkeA2 } \\
\text { (pke) }\end{array}$ & Missense (F1282V) & $\begin{array}{l}\text { Modular polyketide synthase (module 1: KS-AT(A)-DH-KR-ACP; module 2: KS-AT(A)- } \\
\text { DH-KR-ACP) }\end{array}$ \\
\hline SACE_5308 & 1730 & (pks7) & Missense (A795T) & Iterative type 1 polyketide synthase \\
\hline
\end{tabular}

Non-ribosomal peptide synthases and related biosynthetic proteins

\begin{tabular}{lllll}
\hline SACE_3015 & 1083 & (nrps4) & 1PM & Non-ribosomal peptide synthase \\
\hline SACE_3016 & 334 & (nrps4) & Missense (V245F) & SyrP-like protein \\
\hline SACE_3033 & 441 & & Missense (R186C) & Lysine/ornithine N-monooxygenase \\
\hline SACE_3057 & 547 & & Missense (A481T) & 2-polyprenyl-6-methoxyphenol hydroxylase \\
\hline SACE_4288 & 7259 & (nrpsl) & Missense (P6258L) & Non-ribosomal peptide synthase \\
\hline
\end{tabular}

Terpene synthases and related biosynthetic proteins

\begin{tabular}{lllll}
\hline SACE_3187 & 758 & $\begin{array}{l}\text { (tpcl) } \\
\text { geol) }\end{array}$ & Nonsense (W83*) & Terpene synthase metal-binding domain-containing protein \\
\hline SACE_3978 & 466 & $\begin{array}{lll}(\text { tpc3/ } \\
\text { geo2) }\end{array}$ & $\begin{array}{l}\text { Frameshift }(+\mathrm{G} \\
1040 / 1041)\end{array}$ & Cyclic nucleotide-binding domain-containing protein (tpc3 gene cluster) \\
& & &
\end{tabular}

a IPM, imperfect match

Abbreviations: $C L$ thioester ligase; $K S Q$ Initiating decarboxylase; $K S$ ketosynthase; $A T$ acyltransferase (A and $\mathrm{P}$ refer to specificity for acetate and propionate units respectively); $A C P$ acyl carrier protein; $K R$ ketoreductase; $T E$ thioesterase; $A M T$ aminotransferase; $P 450$ cytochrome $P 450$ hydroxylase; $D H$ dehydratase; $E R$ enoylreductase

coding for an unknown type II polyketide [23], which was up-regulated in Px strain during phase a. Moreover, while in NRRL 2338 the biosynthetic clusters nrps3, $n r p s 5$, tpc2, tpc3 and tpc4 clearly appeared to be downregulated during phase a, in Px only two clusters, tpc4 and $t p c 5$, exhibited this behavior suggesting profound differences in control of secondary metabolism between the two strains (Figure 5A and 5B). The LAP analysis failed to identify regions exhibiting significant differential gene expression between phase $\mathbf{b}$ and phase $\mathbf{c}$ in NRRL 2338 see Peano et al. 2007 [24].

\section{Central carbon and nitrogen metabolism in S. Erythraea px}

Table 1 shows that a considerable number of mutated genes are involved in central carbon (and energy) and nitrogen metabolism, or related to substrate uptake and utilization (Table 2). This is not surprising because erythromycin biosynthesis is strictly connected with central metabolism consistently with both genomic and expression data. In general, secondary metabolism is believed dispensable for survival, and most of gene clusters coding for secondary metabolites occupy non-core genomic regions and are maximally expressed during late growth phases. In contrast, the ery cluster maps in the core 
Table 4 Variations within genes related to transcription and translation processes

\begin{tabular}{|c|c|c|c|c|}
\hline $\begin{array}{l}\text { Gene locus in } \\
\text { NRRL } 2338\end{array}$ & $\begin{array}{l}\text { Length } \\
\text { (amino } \\
\text { acids) }\end{array}$ & $\begin{array}{l}\text { Gene } \\
\text { (locus) }\end{array}$ & Variation $^{\mathrm{a}}$ & Protein function and notes \\
\hline SACE_2076 & 148 & nusB & $\begin{array}{l}\text { Frameshift } \\
(-G \text { 316) }\end{array}$ & NusB antiterminator factor No paralogue. \\
\hline \multicolumn{5}{|c|}{ Transcription factors } \\
\hline SACE 0891 & 206 & & Frameshift (+G 581/582) & TetR family transcriptional regulator \\
\hline SACE_1040 & 163 & & Missense (R30C) & MarR family transcriptional regulator \\
\hline SACE_1895 & 281 & & Frameshift (+C 603/604) & XRE family transcriptional regulator \\
\hline SACE_2927 & 918 & & Missense (P52L) & SARP family transcriptional regulator \\
\hline SACE_3079 & 294 & & $\begin{array}{l}\text { Frameshift } \\
(-C 506)\end{array}$ & LysR family transcriptional regulator \\
\hline SACE_3348 & 96 & & Missense (E60A) & XRE family transcriptional regulator \\
\hline SACE_4500 & 233 & & $\begin{array}{l}\text { Frameshift } \\
(-C \text { 129; }-G \text { 222) }\end{array}$ & GntR family transcriptional regulator \\
\hline SACE_4536 & 327 & & Missense (T246N, L249M) & Lacl family transcription regulator \\
\hline$\overline{\text { SACE_4775 }}$ & 246 & & Missense (A96V) & MerR family transcriptional regulator \\
\hline SACE_5410 & 287 & & Missense (P140F) & XRE family transcriptional regulator \\
\hline SACE_5425 & 505 & & $\begin{array}{l}\text { Missense and in frame deletion (V297S, V298W, A299R, } \\
\text { V300C, Q301R A302P, H303R, D304-, G305R) }\end{array}$ & PucR- family transcriptional regulator \\
\hline $\begin{array}{l}\text { SACE_5448- } \\
\text { SACE_5449 }\end{array}$ & $\begin{array}{l}126 \\
150\end{array}$ & & Duplication of SACE_5448 and SACE_5449 & $\begin{array}{l}\text { SACE_5448: Unknown function SACE 5449: LuxR } \\
\text { family transcriptional regulator }\end{array}$ \\
\hline SACE_5739 & 282 & & Missense (K206T) & XRE family transcriptional regulator \\
\hline$\overline{\text { SACE_6021 }}$ & 484 & & Frameshift (+A 1212/1213) & PucR- family transcriptional regulator \\
\hline \multicolumn{5}{|c|}{ Signal transduction } \\
\hline SACE_1833 & 241 & & Frameshift (-C 667) & Two-component system response regulator \\
\hline$\overline{\text { SACE_1879 }}$ & 587 & & Frameshift (+C 1002/1003) & Serine/threonine protein kinase \\
\hline SACE_1988 & 150 & & Missense (G125D) & Universal stress protein UspA \\
\hline SACE_2583 & 541 & & Nonsense (R478*) & $\mathrm{N}$-acyl D-amino acid deacylase \\
\hline SACE_4937 & 414 & & Missense (G30R) & Mandelate racemase/starvation sensing protein \\
\hline SACE_5284 & 286 & stap & IPM & $\begin{array}{l}\text { Endonuclease/Exonuclease/Phosphatase family } \\
\text { protein }\end{array}$ \\
\hline SACE_5286 & 224 & & Frameshift (-G419, -G 430) & Two-component system response regulator \\
\hline SACE_5301 & 533 & & Frameshift (+C 1585/1586) & N-acyl_D-amino acid deacylase \\
\hline SACE 6086 & 879 & $k d p D$ & Missense (P828L) & Osmosensitive K+ channel histidine kinase \\
\hline SACE_6447 & 228 & $m t r A$ & In frame deletion (VI96-, HI97-) & Two-component system response regulator \\
\hline SACE_6490 & 424 & & $\begin{array}{l}\text { Missense } \\
\text { (S37R) }\end{array}$ & Two-component system sensor kinase \\
\hline SACE_6720 & 526 & phoD & Missense (C328Y, P382F) & $\begin{array}{l}\text { Phosphodiesterase/alkaline phosphatase D } \\
\text { (phosphate starvation) }\end{array}$ \\
\hline SACE_7263 & 382 & & Missense (A222T) & Two-component system sensor kinase \\
\hline \multicolumn{5}{|c|}{ Translation machinery } \\
\hline$\overline{\text { SACE_0443 }}$ & 464 & cysS & Missense (G198S) & Cysteinyl-tRNA synthetase \\
\hline$\overline{\text { SACE_0799 }}$ & 593 & mets & Missense (P569S) & Methionyl-tRNA synthetase \\
\hline SACE_2075 & 188 & efp & Frameshift (+C 504) & Elongation factor $P$ \\
\hline$\overline{\text { SACE_3403 }}$ & 474 & gatA & Missense (A243E) & $\begin{array}{l}\text { Asp-tRNAAsn/Glu-tRNAGln amidotransferase A } \\
\text { subunit }\end{array}$ \\
\hline SACE_5926 & 1027 & $\operatorname{infB}$ & Missense (A278V) & Translation initiation factor 2 \\
\hline SACE_5919 & 536 & & Frameshift (-G 821) & Pseudouridine synthase \\
\hline \multicolumn{5}{|c|}{ Protein turnover and chaperones } \\
\hline SACE_1339 & 860 & & IPM & Aminopeptidase N \\
\hline
\end{tabular}


Table 4 Variations within genes related to transcription and translation processes (Continued)

\begin{tabular}{lllll}
\hline SACE_2951 & 174 & ClpC & Frameshift (+G 468/469) & $\begin{array}{l}\text { ATPases with chaperone activity, ATP-binding } \\
\text { subunit }\end{array}$ \\
\hline SACE_3756 & 768 & ClpA & Missense (I284T) & $\begin{array}{l}\text { ATPases with chaperone activity, ATP-binding } \\
\text { subunit }\end{array}$ \\
\hline SACE_6784 & 610 & & Frameshift (-C 1573) & Molecular chaperone \\
\hline SACE_6113 & 481 & IPM? & Aminopeptidase \\
\hline
\end{tabular}

IPM, imperfect match

region of the S. erythraea chromosome, and is transcribed during the middle pseudo-exponential growth phase when the activities if the carbon and nitrogen central metabolic pathways are maximal (Figures 4 and 5) $[23,24,32,36]$.
As shown in Figure 6, these pathways are strictly connected to erythromycin biosynthesis that requires one propionyl-CoA and six (2S)-methylmalonyl-CoA units for assembly of the 14- membered macrolactone 6DEB. Different metabolic routes may accomplish

Table 5 Variations within genes related to cell division, DNA replication and repair, transposition and phage integration

\begin{tabular}{|c|c|c|c|c|}
\hline $\begin{array}{l}\text { Gene locus in } \\
\text { NRRL } 2338\end{array}$ & $\begin{array}{l}\text { Length } \\
\text { (amino } \\
\text { acids) }\end{array}$ & $\begin{array}{l}\text { Gene } \\
\text { (locus) }\end{array}$ & Variation $^{\mathrm{a}}$ & Protein function and notes \\
\hline \multicolumn{5}{|c|}{ DNA replication and repair } \\
\hline SACE_0826 & 1195 & $m f d$ & Frameshift (+A 218/219) & Transcription/repair coupling factor \\
\hline SACE_1351 & 269 & $\begin{array}{l}\text { mutM, } \\
\text { fpg }\end{array}$ & Missense (M199T) & Formamidopyrimidine DNA glycosylase \\
\hline SACE_3677 & 147 & ogtl & Missense (G41K) & Methylated DNA-protein cysteine methyltransferase \\
\hline SACE_4427 & 183 & & Frameshift (+G 353/354) & G/U mismatch-specific DNA glycosidase \\
\hline SACE_5437 & 895 & polA & Frameshift (-C617) & DNA polymerase I \\
\hline SACE_5255 & 207 & alkA & Missense (P68F) & 3-methyladenine DNA glycosylase \\
\hline \multirow[t]{2}{*}{ SACE_6108 } & 693 & uvrD2 & IPM & ATP-dependent DNA helicase UvrD-like \\
\hline & & & Missense and in & \\
\hline SACE_6681 & 873 & uvrD & frame deletion (G54-, G56R, S57I) & ATP-dependent DNA helicase UvrD \\
\hline \multicolumn{5}{|l|}{ Cell division } \\
\hline SACE_0667 & 941 & & Missense (F874L) & DNA segregation ATPase FtsK/SpolllE \\
\hline SACE_6104 & 263 & & IPM & Cell division initiation protein \\
\hline SACE_6929 & 579 & & Missense (P159R) & ATPase involved in chromosome partitioning \\
\hline \multicolumn{5}{|c|}{ Transposition and phage integration } \\
\hline SACE_0657 & 106 & & Excision & IS1647-like transposase \\
\hline SACE_2154 & 350 & & IPM & Transposase, IS891/IS1136/IS1341 \\
\hline $\begin{array}{l}\text { SACE_2214and } \\
\text { similar CDSs }\end{array}$ & 365 & & $\begin{array}{l}\text { Insertion between SACE_4827 and } \\
\text { SACE_4228 }\end{array}$ & $\begin{array}{l}\text { SACE-2214: Transposase SACE_4827: Oxidoreductase } \\
\text { SACE_4228: Lacl family transcriptional regulator }\end{array}$ \\
\hline $\begin{array}{l}\text { SACE_2313 and } \\
\text { similar CDSS }\end{array}$ & 99 & & $\begin{array}{l}\text { Insertion between SACE_2371 and } \\
\text { SACE_2372 }\end{array}$ & $\begin{array}{l}\text { SACE_2313: IS4-like transposase SACE_2371: IS4-like } \\
\text { transposase SACE_2372: IS 1647-like transposase }\end{array}$ \\
\hline SACE_2322 & 374 & & Missense (VI391) & Phage-related integrase/Site-specific recombinase XerC \\
\hline SACE_3579 & 469 & $\operatorname{tnp}$ & Missense (R13S, A73S, T118A, V309A) & Transposase, 1S1XX5 \\
\hline SACE_4072 & 469 & $\operatorname{tnp}$ & $\begin{array}{l}\text { Missense (S178L, Q191R, S199A,H218R, } \\
\text { N370T, G430S, T431V) }\end{array}$ & Transposase, ISIxx5 \\
\hline \multirow[t]{2}{*}{ SACE_5073 } & 459 & & Missense (N171K) & Transposase, IS3508i \\
\hline & & & Missense and in frame insertion & \\
\hline SACE_5268 & 395 & & $\begin{array}{l}\text { (S73T, A74P, H129N,-134T, -135V,L191V, } \\
\text { I216V A296V, P388R, S396L) }\end{array}$ & Transposase inactivated by frameshift mutation) \\
\hline SACE_5430 & 232 & & Missense (H15R, R193W) & Transposase, Tn5714 \\
\hline
\end{tabular}

aPM, imperfect match 
Table 6 Variations within genes of unknown function

\begin{tabular}{|c|c|c|c|}
\hline $\begin{array}{l}\text { Gene } \\
\text { locus in } \\
\text { NRRL }\end{array}$ & $\begin{array}{l}\text { Length } \\
\text { (amino } \\
\text { acids) }\end{array}$ & $\begin{array}{l}\text { Gene/ Variation } \\
\text { locus }\end{array}$ & Protein function and notes \\
\hline
\end{tabular}

2338

Unknown function

SACE $0062 \quad 35$

Insertion betweenSACE_ 2351 and SACE_2352

SACE_0062: Unknown function. SACE_2351: Tn5714like transposase. SACE_2352: IS 111 a/IS 1328/IS1533like transposase.

\begin{tabular}{|c|c|c|c|}
\hline SACE_0062 & 35 & $\begin{array}{l}\text { Insertion in SACE_5430, between SACE_5429 and } \\
\text { SACE_5431 }\end{array}$ & $\begin{array}{l}\text { SACE_0062: Unknown function. SACE_5429: } \\
\text { Dephospho-CoA kinase SACE_5430: Transposase, } \\
\text { Tn5714 SACE_5431: 30S ribosomal protein SI }\end{array}$ \\
\hline SACE_0157 & 389 & Missense (P295L) & Unknown function/Glutathionylspermidine synthase \\
\hline SACE_0587 & 995 & Frameshift (+G 332/333) & Unknown function \\
\hline SACE_0744 & 164 & Missense (R42H) & Unknown function \\
\hline SACE_0940 & 289 & IPM & Unknown function \\
\hline SACE_0944 & 831 & Nonsense $\left(Y 412^{*}\right)$ & Unknown function \\
\hline SACE_1128 & 294 & Missense (VI13 A) & Unknown function \\
\hline SACE_1129 & 774 & Missense (A172T) & Unknown function \\
\hline SACE_1257 & 195 & Nonsense $\left(\mathrm{Q} 60^{*}\right)$ & $\begin{array}{l}\text { Unknown function/uncharacterized MobA-related } \\
\text { protein }\end{array}$ \\
\hline SACE_1344 & 1638 & IPM? & Unknown function/PE-PGRS family protein \\
\hline SACE_1805 & 100 & Missense (S13F) & Unknown function \\
\hline SACE_1835 & 175 & IPM & Unknown function \\
\hline SACE_1853 & 110 & Frameshift (+G 51/52) & Unknown function \\
\hline SACE_2384 & 496 & Frameshift (+T 823/824) & Unknown function \\
\hline SACE_2456 & 251 & Missense (A28V) & Unknown function \\
\hline$\underline{\text { SACE_2737 }}$ & 116 & Missense (P4R) & Unknown function \\
\hline SACE_3005 & 316 & Frameshift (-G 727) & Unknown function \\
\hline$\underline{\text { SACE_3102 }}$ & 1249 & Frameshift (+G 338/339) & Unknown function \\
\hline SACE_3186 & 392 & Missense (R390S) & Unknown function (tpcl cluster) \\
\hline SACE_3262 & 282 & Frameshift (+C 434/435) Insertion between & $\begin{array}{l}\text { Unknown function } \\
\text { SACE 3264: Unknown secreted protein. }\end{array}$ \\
\hline SACE_3264 & 117 & SACE 3572 and SACE 3573 & $\begin{array}{l}\text { SACE 3572: Unknown secreted protein. SACE 3573: } \\
\text { Unknown function }\end{array}$ \\
\hline$\underline{\text { SACE_3748 }}$ & 277 & Frameshift (+C 696/697) & Unknown function \\
\hline SACE_3850 & 261 & Frameshift (-G 388) & Unknown function \\
\hline$\underline{\text { SACE_3925 }}$ & 188 & Frameshift (+A 437/438) & Unknown function \\
\hline SACE_4249 & 234 & Frameshift (-G 640) & Unknown function \\
\hline SACE_4310 & 480 & $\begin{array}{l}\text { Frameshift } \\
(- \text { C 1146) }\end{array}$ & Unknown function \\
\hline SACE_4451 & 291 & In frame insertion $(-102 \mathrm{D},-103 \mathrm{~A},-104 \mathrm{~N},-105 \mathrm{E},-106 \mathrm{Q})$ & Unknown function \\
\hline SACE_4989 & 38 & Insertion between SACE_5103 and SACE_5105 & $\begin{array}{l}\text { SACE_4989: Unknown function. SACE_5103: Type III } \\
\text { restriction enzyme, res subunit. } \\
\text { SACE_5105: Transposase }\end{array}$ \\
\hline SACE_5311 & 835 & Missense (G411R) Frameshift & Unknown function \\
\hline SACE_5423 & 184 & $(-\mathrm{G} 372,-\mathrm{G} 473,-\mathrm{C} 481,-\mathrm{G} 493,-\mathrm{T}$ 409,-C 540) & Unknown function \\
\hline SACE_5446 & 1184 & Frameshift (-C 2898) & Unknown function \\
\hline SACE_5460 & 330 & Missense (P162F) & Unknown function \\
\hline SACE_5482 & 632 & $\begin{array}{l}\text { Missense } \\
\text { (L509P) }\end{array}$ & Unknown function \\
\hline SACE_5483 & 11792 & Missense (L1329F, T5002A, P8537L, R9518H) & Unknown function \\
\hline
\end{tabular}


Table 6 Variations within genes of unknown function (Continued)

\begin{tabular}{|c|c|c|c|}
\hline SACE_5513 & 566 & $\begin{array}{l}\text { In frame deletion (S249-, E250-,G251-, T252-,T253-, G254-, } \\
\text { G255-, T256-, G257-, G258-, A259-, G260-) }\end{array}$ & Unknown function \\
\hline SACE_5523 & 5856 & Nonsense (L370*) & Unknown function \\
\hline SACE_5655 & 31 & Duplication of SACE_5655 & Unknown function \\
\hline SACE_5905 & 418 & $\begin{array}{l}\text { Missense and in frame insertion (A5V, T7G, I8C, R10H, V11H, } \\
\text { Q12L, T13A, M14G, S15A I16D, E17H, S18E, A19H, R21E, } \\
\text { T22R, L24A, -25H, -26R, -27A) }\end{array}$ & Unknown function \\
\hline SACE_6184 & 171 & Frameshift (-G 169) & Unknown function \\
\hline SACE_6567 & 206 & $\begin{array}{l}\text { Missense, in frame insertion, nonsense (A135G,-136R,- 137R,- } \\
\text { 138G,-139A,- 140A,-141R,-142W, -143G,-144P, -145P, -146R,-- } \\
\text { 1475,-148*, A149R, L155R, G156A, T157R, A158-, A159-, V160- } \\
\text {, V161-, T161-, F163-, A165*, A166R,I167T, A168G, T170-, } \\
\text { V171-) V172-,K173-,D174-, W175-,F176-, V177-, A178R, A164S }\end{array}$ & Unknown function \\
\hline SACE_6773 & 962 & Missense (D330E) Frameshift (-G 1654) & Unknown function/PE-PGRS family protein \\
\hline SACE_7193 & 278 & Frameshift (+C 729/730) & Unknown function \\
\hline SACE_7240 & 265 & Missense (P9OH) & Unknown function \\
\hline SACE_7316 & 47 & Duplication of SACE_7316 & Unknown function \\
\hline
\end{tabular}

IPM, imperfect match

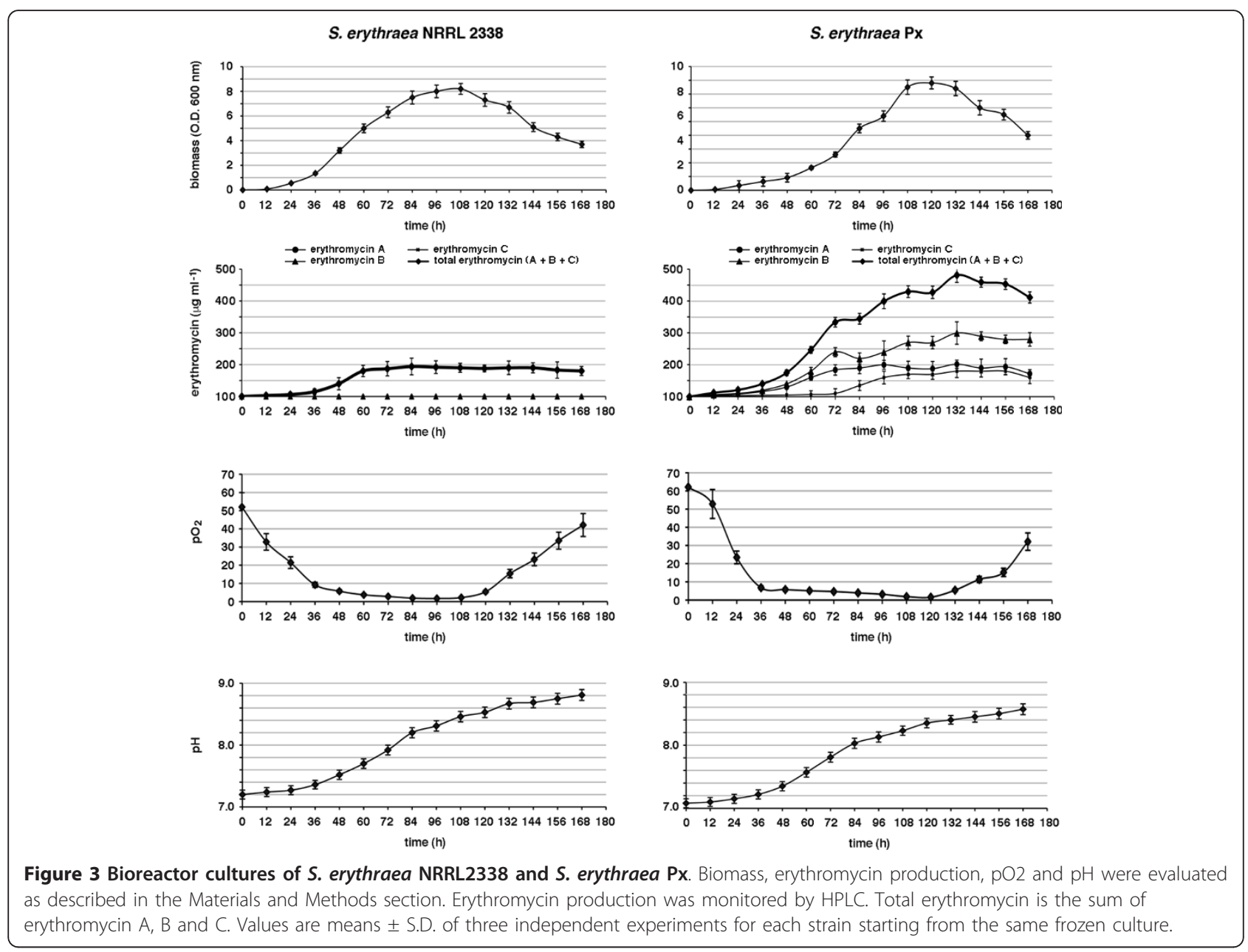




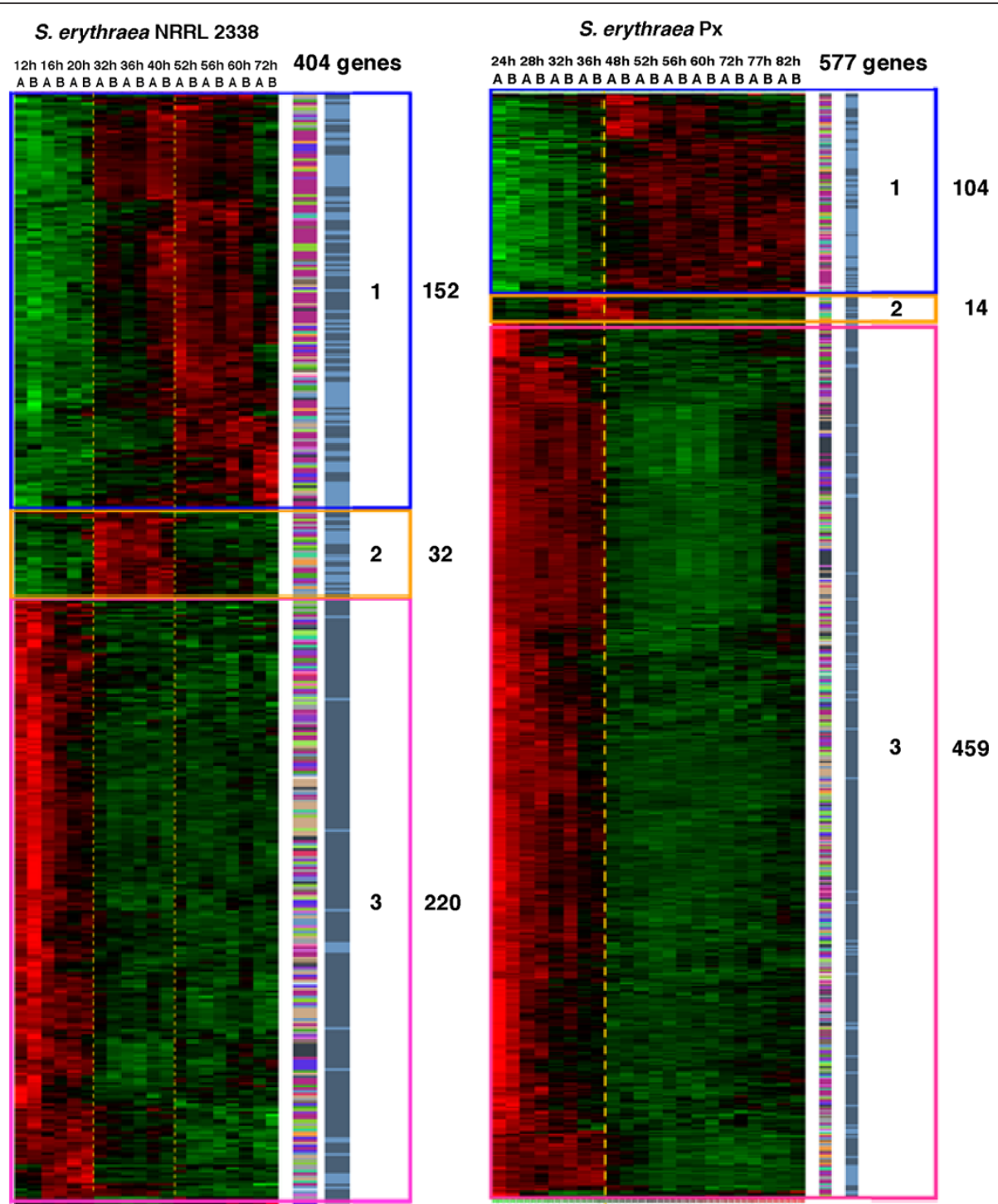

Figure 4 Microarray analysis showing changes in global control of cell-cycle. Visualization by dChip of 404 S. erythraea NRRL2338 and of 577 S. erythraea Px differentially expressed genes, selected by a q-value $<=0,001$, and determining a time course gene expression profiling and a hierarchical clustering of the samples (Red = up-regulation; Green = down-regulation); on the right side of the figure, two columns respectively show the functional classification of the DEGs whose transcription profile is visualized and their positional distribution in the core (dark blue) and non core region (light blue) of the genome.

precursor supply for erythromycin biosynthesis. In addition to well-established propanoate metabolism leading to both propionyl-CoA and six (2S)-methylmalonyl-CoA units, alternative routes exist including valine, leucine and isoleucine degradation pathways, and glycine, serine and threonine metabolism. An additional proposed route to (2S)-methylmalonyl-CoA proceeds by the rearrangement of succinyl-CoA catalyzed by methylmalonyl-CoA mutase yielding the (2R)isomer of methylmalonyl-CoA that would be converted to the (2S)- isomer by a methylmalonyl-CoA epimerase.
Our analysis demonstrated that the several genes coding for the component enzymes of the tricarboxylic acid cycle (TCA) were mutated in Px strain. In particular, pyc gene (SACE_6118) coding for pyruvate carboxylase (PC) was inactivated by a frameshift mutation. This result is noteworthy, because PC serves a major anaplerotic role for the TCA by catalyzing the ATP-dependent carboxylation of pyruvate to oxalacetate, and there is evidence that in Corynebacterium glutamicum PC contributes about $90 \%$ to $C(3)$ carboxylation at the anaplerotic node (Petersen, 2000). Moreover, pyc has no obvious paralog(s) in the genome of S. erythraea. In 
Table 7 Functional classification of cell cycle-regulated genes in both strains ${ }^{a}$

\begin{tabular}{|c|c|c|c|c|}
\hline & & Strain & NRRL2338 & Px \\
\hline COG & Functional categories & $\begin{array}{c}\% \text { COG } \\
\text { probesets }\end{array}$ & $\begin{array}{l}\text { \% Probesets with q-value }<= \\
0.001\end{array}$ & $\begin{array}{l}\% \text { Probesets with q-value }<= \\
0.001\end{array}$ \\
\hline $\bar{I}$ & Cellular processes and signaling & & & \\
\hline 1.1 & $\begin{array}{l}\text { Cell cycle control, cell division, chromosome } \\
\text { partitioning }\end{array}$ & 0,35 & 0,99 & 0,69 \\
\hline 1.2 & Cell motility & 0,01 & 0 & 0 \\
\hline 1.3 & Cell wall/membrane/envelope biogenesis & 2,05 & 3,22 & 3,81 \\
\hline 1.4 & Defense mechanisms & 0,89 & 0,00 & 0,87 \\
\hline 1.5 & $\begin{array}{l}\text { Intracellular trafficking, secretion, and vesicular } \\
\text { transport }\end{array}$ & 0,24 & 0,25 & 0,69 \\
\hline 1.6 & $\begin{array}{l}\text { Posttranslational modification, protein turnover, } \\
\text { chaperones }\end{array}$ & 1,90 & 5,94 & 3,99 \\
\hline 1.7 & Signal transduction mechanisms & 1,91 & 0,99 & 1,91 \\
\hline II & Information storage and processing & & & \\
\hline 11.1 & Amino acid transport and metabolism & 0,01 & 0 & 0 \\
\hline 11.2 & Cell wall/membrane/envelope biogenesis & 0,01 & 0 & 0 \\
\hline 11.3 & Chromatin structure and dynamics & 0,01 & 0 & 0 \\
\hline 11.4 & General function prediction only & 0,30 & 0,50 & 0,35 \\
\hline 11.5 & Nucleotide transport and metabolism & 0,07 & 0 & 0 \\
\hline$\overline{11.6}$ & $\begin{array}{l}\text { Posttranslational modification, protein turnover, } \\
\text { chaperone }\end{array}$ & 0,04 & 0 & 0 \\
\hline 11.7 & Replication, recombination and repair & 2,44 & 0,74 & 2,43 \\
\hline 11.8 & RNA processing and modification & 0,01 & 0 & \\
\hline 11.9 & $\begin{array}{l}\text { Secondary metabolites biosynthesis, transport } \\
\text { catabolism }\end{array}$ & 0,04 & 0 & \\
\hline 11.10 & Signal transduction mechanisms & 0,71 & 0,50 & 0,69 \\
\hline 11.11 & Transcription & 7,20 & 2,23 & 3,29 \\
\hline 11.12 & Translation, ribosomal structure and biogenesis & 2,48 & 4,21 & 10,75 \\
\hline III & Metabolism & & & \\
\hline$\overline{111.1}$ & Amino acid transport and metabolism & 6,60 & 0 & 8,15 \\
\hline 111.2 & Carbohydrate transport and metabolism & 6,08 & 7,67 & 6,59 \\
\hline 111.3 & Cell wall/membrane/envelope biogenesis & 0,38 & 0 & 0,17 \\
\hline$\overline{111.4}$ & Coenzyme transport and metabolism & 2,63 & 5,20 & 4,33 \\
\hline 111.5 & Energy production and conversion & 4,79 & 9,90 & 8,15 \\
\hline 111.6 & Inorganic ion transport and metabolism & 2,68 & 4,21 & 2,25 \\
\hline 111.7 & Lipid transport and metabolism & 4,24 & 2,72 & 5,03 \\
\hline 111.8 & Nucleotide transport and metabolism & 1,27 & 2,97 & 3,64 \\
\hline 111.9 & $\begin{array}{l}\text { Posttranslational modification, protein turnover, } \\
\text { chaperones }\end{array}$ & 0,08 & 0 & 0,17 \\
\hline 111.10 & $\begin{array}{l}\text { Secondary metabolites biosynthesis, transport } \\
\text { catabolism }\end{array}$ & 2,90 & 2,48 & 2,77 \\
\hline $\begin{array}{l}111 . \\
11\end{array}$ & Signal transduction mechanisms & 0,16 & 0 & 0 \\
\hline IV & Poorly characterized & & & \\
\hline IV.1 & Function unknown & 32,11 & 27,23 & 22,70 \\
\hline \multirow[t]{2}{*}{$\mathrm{IV} .2$} & General function prediction only & 7,37 & 8,17 & 6,59 \\
\hline & Total probesets & 7060 & 404 & 577 \\
\hline
\end{tabular}

aThe categories over-represented and under-represented are evidenced, respectively, by BOLD and oblique number 
A

A. erythraea NRRL 2338
phase a vs phase b

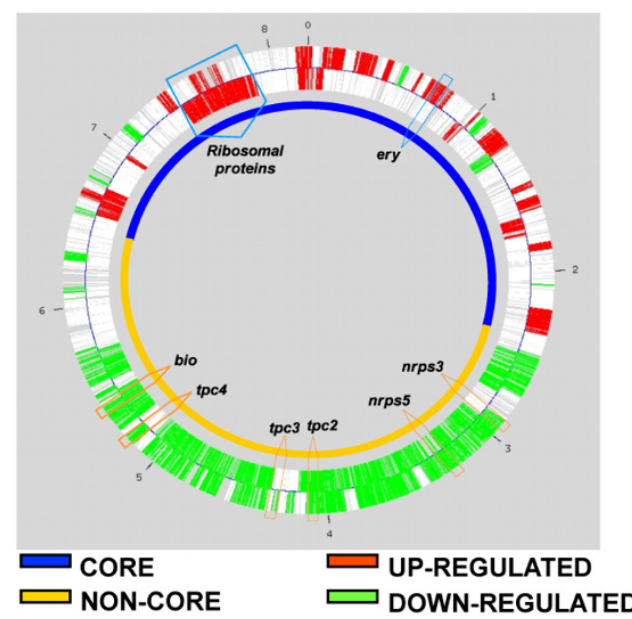

B

S. erythraea Px

phase a vs phase b

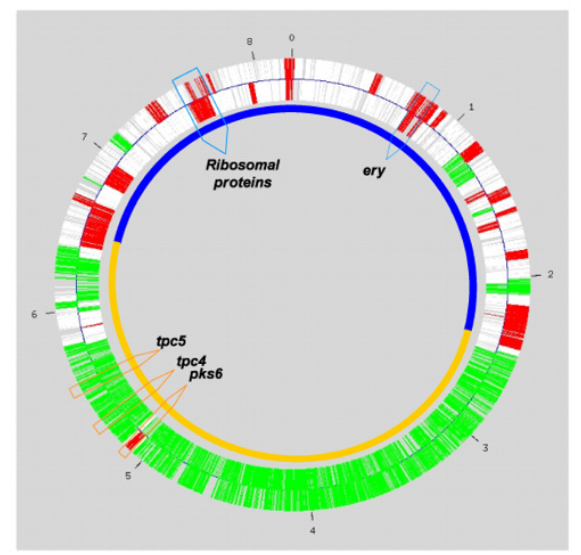

Figure 5 Visualization of the LAP algorithm results on the two chromosome strands independently analyzed. Comparison between the transcriptional profiling of all the 6494 S. erythraea genes in phase A versus phase B in the NRRL2338 strain(A) and in the Px strain(B) is shown. A q value of 0,01 and a fold change of 0,5 were chosen as filtering parameters. The transcriptionally up-modulated regions are shown in RED while the down- modulated are in GREEN. The OriC is indicated as $\mathrm{O}$ and the resolution of the chromosome is $1 \mathrm{Mb}$. The core region is evidenced in blue and the non-core region in orange; the position of clusters involved in the secondary metabolism, erythromycin production and coding for ribosomal proteins are outlined by arrows.

addition to $p y c$, non-conservative missense mutations affected both the E1 and the E2 component-encoding genes (sucA [SACE_6385] and sucB [SACE_1638], respectively) of the 2-oxoglutarate dehydrogenase complex. 2- oxoglutarate dehydrogenase is a key enzyme in the TCA cycle, converting 2-oxoglutarate, coenzyme $\mathrm{A}$ and $\mathrm{NAD}(+)$ to succinyl-CoA, NADH and carbon dioxide. This activity is tightly regulated and it is a

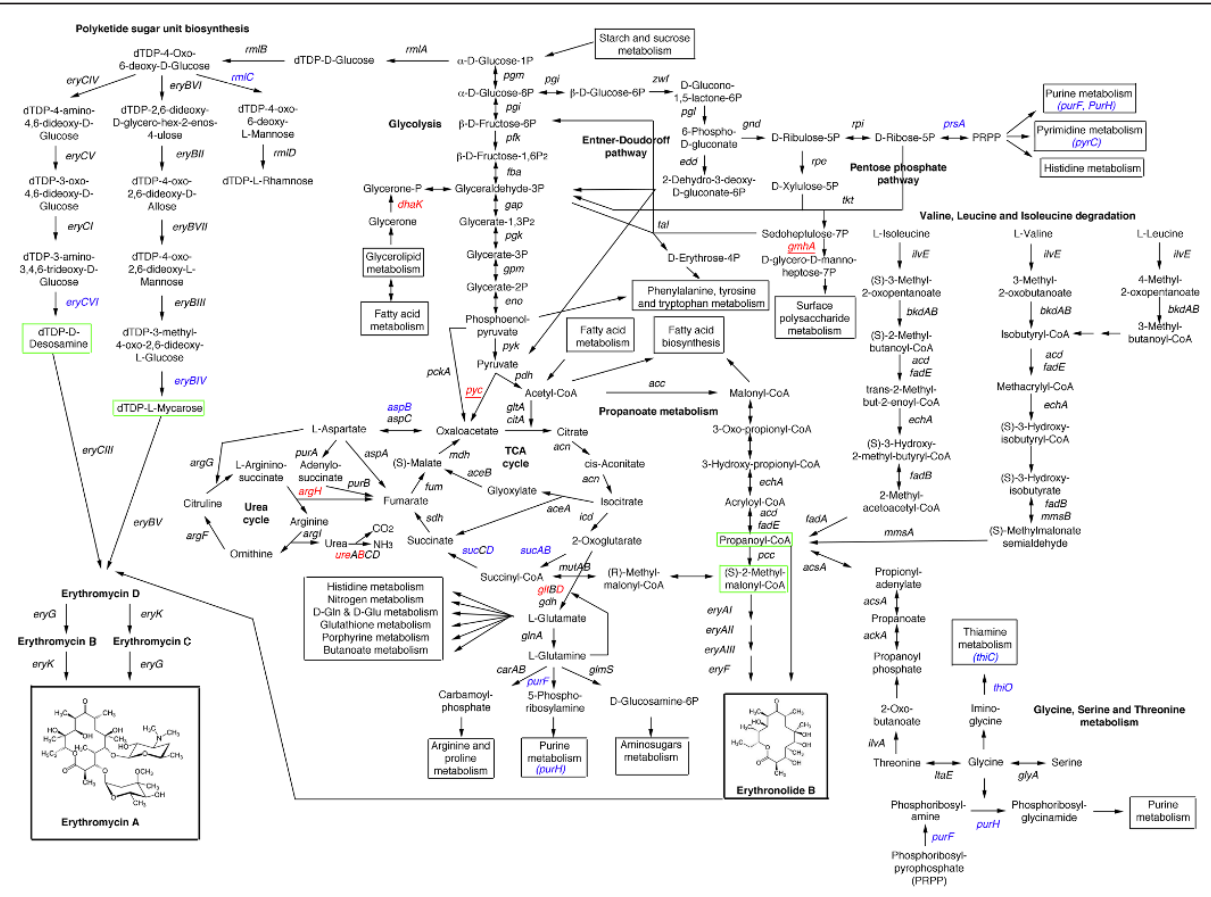

Figure 6 Overview of carbon and nitrogen metabolic pathways and their relationships with the biosynthesis of erythromycin. Genes affected by missense mutations are indicated in blue; genes affected by missense/nonsense mutations are shown in red. Single-copy genes are underlined. 
major determinant of the metabolic flux through the TCA cycle. Also in this case, obvious paralogs could not be found in the genome of this microorganism. Although it is difficult to predict the effect of the observed mutations on the activity of the 2- oxoglutarate dehydrogenase complex, altogether our results are consistent with the hypothesis that the carbon flux through the TCA cycle may be greatly reduced in the Px strain, and that, as a consequence, more acetyl-CoA is funneled into alternative routes including propanoate metabolism leading to the 6DEB precursors (Figure 6).

This view is supported by evidence that glutamine synthatase/glutamate synthase (GS- GOGAT) and urea cycles, which are linked to TCA by 2-oxoglutarate and oxaloacetate/fumarate, respectively, may also be affected in the Px strain. Indeed, in this strain, gltD, coding for small subunit of glutamate synthase (SACE_5741), a component of the high-affinity ammonium assimilation system, is inactivated by a frameshift mutation (Table 1 and Figure 6). However, in this case, a paralogous gene (SACE_3997) is predicted to exist in the genome, to alleviate the deleterious effect of such a mutation. A possible deficiency in the high-affinity ammonium assimilation system may also account for the different behavior of S. erythraea NRRL 2338 and Px strain with respect to ammonium content in the growth medium. Growth, pigment and antibiotic production was strongly inhibited in the wild type strain in the presence of high ammonium content, while, in contrast, the same parameters were not affected by ammonium in the erythromycin over-producing strain (Figure 7). Frameshift mutations inactivate SACE_6330 (argH domain) and SACE_0635 (ureB). These CDSs may be involved in the urea cycle as they code, respectively, for a fusion protein with both ligase/carboxylase and argininosuccinate lyase domains, and urease beta subunit. In the latter case, no obvious $u r e B$ paralog could be detected in the genome. In addition to the urea cycle, one of the two aspartate aminotransferase-encoding paralog (aspB) (SACE_4319), whose activity is linked to the urea cycle and is essential for growth on L-glutamate, was also affected by a nonconservative missense mutation.

In the absence of sulfate and cysteine, several bacteria can use aliphatic sulfonates as a source of sulfur for growth. tau $A B C D$ genes coding for an ABC-type transport system required for uptake of aliphatic sulfonates and a desulfonation enzyme accomplish this property. In Px strain tauC (SACE_4434) coding for permease component of taurine transport system, and tauD (SACE_4651) coding for 2-oxoglutarate-dependent taurine dioxygenase are affected by missense mutations, along with cdo2 (SACE_6133) encoding a cysteine dioxygenase, which catalyzes the conversion of L-cysteine to cysteine sulfinic acid, a compound that lies at a branch-
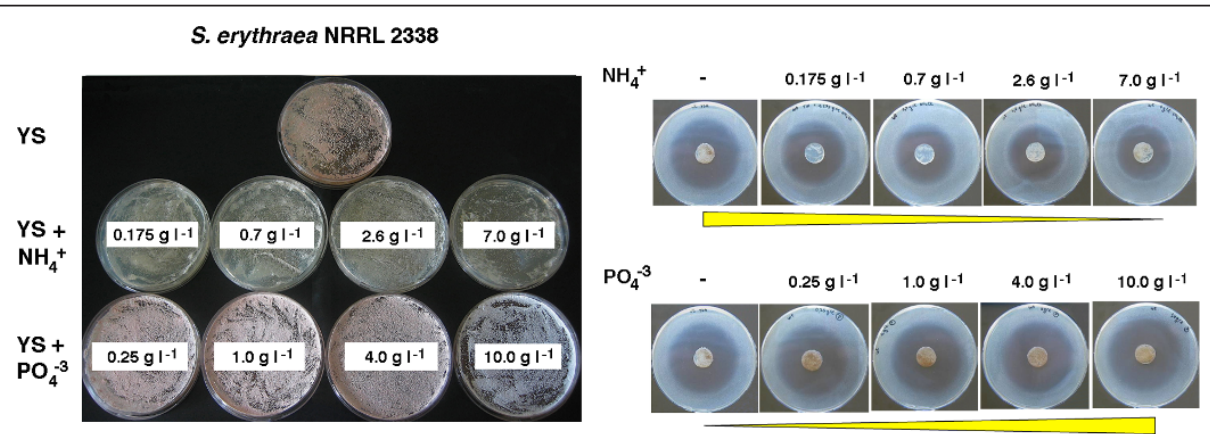

S. erythraea Px
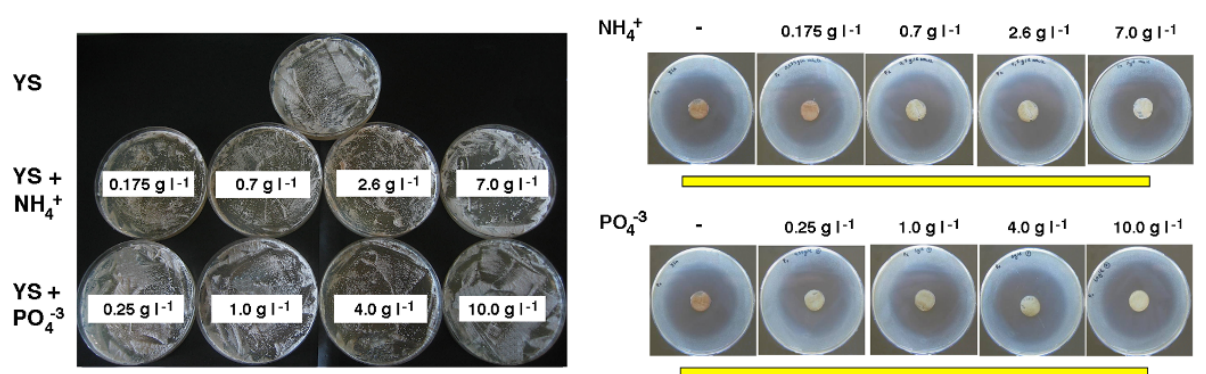

Figure 7 Effect of ammonia and phosphate on growth, pigmentation, sporulation and antibiotic production. S. erythraea NRRL 2338 and S. erythraea Px were cultivated for 7 days on YS agar supplemented with the indicated ammonia and phosphate concentrations (left panels). Antibiotic production in these media was evaluated by microbiological assay using Micrococcus luteus as a tester organism (right panel) 
point in cysteine catabolism, where it can follow two pathway resulting in the formation of taurine or sulfate (Table 1). Down-modulation or inactivation of this metabolic pathway involving the 2-oxoglutarate-dependent taurine dioxygenase may be functional to alleviate the reduced carbon flux through the Krebs cycle by increasing the pool of 2- oxoglutarate.

Several genes involved in nucleotide and thiamine metabolism were also affected by non- conservative missense mutations in the $\mathrm{Px}$ strain, including prs $A$ (SACE_2398) coding for phosphoribosylpyrophosphate (PRPP) synthase (which catalyzes the synthesis of the common precursor for biosynthesis of histidine and purine and pyrimidine nucleotides), purF (SACE_7125) and purH (SACE_6664) whose products code for glutamine PRPP amidotransferase and bifunctional phosphoribosylaminoimidazolecarboxamide formyltransferase/IMP cylohydrolase (which catalyzes, respectively, the first and the second steps in the de novo biosynthesis of thiamine and purine nucleotides), thiO (SACE_0506) and thiC (SACE_0511) specifically involved in thiamine biosynthesis, and pyrC (SACE_2080) encoding dihydroorotase, a key enzyme in pyrimidine nucleotide biosynthesis (Table 1 and Figure 6). Mutations in the above-mentioned genes are expected to reduce the carbon flux toward these biosynthetic pathways (purine/thiamine, pyrimidine), and increase the flux toward the pentose phosphate (prs $A$ mutation) and glycine, serine and threonine metabolic pathway (purF, purH, thiO, thiC mutations). The last pathway is a source of precursors for $6 \mathrm{DEB}$ biosynthesis.

The above-mentioned genetic defects in metabolic genes are well correlated with the slow- growth phenotype of the Px strain in mineral medium MM-101, caused by nutritional requirements which are indicative of TCA cycle precursors and PRPP limitations (Additional file 6). At the same time, our results emphasize the balance that must be reached between pathways competing for the same substrate to maintain robustness of the metabolic network.

\section{Secondary metabolism in S. Erythraea px}

In addition to the gene clusters for erythromycin (ery), for a second modular polyketide synthase (PKS) of unknown function $(p k e)$ and for a type III PKS $(r p p A)$, which generates a reddish pigment, the genome sequencing of S. erythraea NRRL 2338 has revealed additional 22 clusters for the biosynthesis of polyketides, terpenes and non-ribosomally synthesized peptides. In Px strain a total of 19 missense/nonsense/frameshift mutations affected genes related to biosynthesis of secondary metabolites (Table 3).

Twelve of them affected genes coding for PKS or related biosynthetic proteins. In particular, a nonsense mutation in SACE_0019 (coding for an ACP), a frameshift mutation in SACE_0022 ( $p f a B$ ) (coding for a modular PKS) and two non-conservative missense mutations in SACE_0023 ( $p f a C)$ (coding for a modular PKS) inactivated the $p f a$ cluster, which appears to govern the biosynthesis of a polyunsaturated fatty acid such as eicosapentaenoic acid. The $p k s 3$ cluster was inactivated by a frameshift mutation in SACE_2875 (coding for a modular PKS) and a non-conservative missense mutations in SACE_2876 $(g d m F)$ (coding for 3-amino-5hydroxybenzoic acid synthase). Missense mutations affected the $p k s 3$-associated SACE_2888 (coding for an aromatic-L-amino acid decarboxylase). Non-conservative missense mutations were mapped in SACE_2630 ( $p k s 2-$ 1) (coding for a modular PKS) of the biosynthetic cluster $p k s 2$, SACE_4140 ( $p k e A 2$ ) (coding for a modular PKS) in the pke cluster, and SACE_5308 coding for a multifunctional single- module PKS enzymes apparently related to the iterative PKSs involved in enediyne or methylsalicylic acid synthesis. It is conceivable that some of these pathways may compete with that of erythromycin for the same substrates and that their inactivation/down-modulation may be beneficial to $6 \mathrm{DEB}$ biosynthesis.

While it is difficult to predict the effects of the missense mutations affecting eryCVI and eryBVI (Table 3) coding for the enzymes catalyzing the last steps of the biosynthesis of dTDP-D-desosamine and dTDP-Lmycarose biosynthesis, the activated sugars that decorate the erythronolide $\mathrm{B}$, the missense mutation affecting SACE_6416 ( $\mathrm{rmlC})$ coding for dTDP-4- dehydrorhamnose 3,5-epimerase (Table 1 and Figure 6) may cause an increase in production of both dTDP-D-desosamine and dTDP-L-mycarose precursors. In fact, the $r m l C$, eryCIV and ery $B V I$ gene products compete for the same substrate (dTDP-4-oxo-6-deoxy-D-glucose). Additional work is required to clarify these aspects, as well as the possible effects of the inactivation/down-modulation of nrps 4 and nrps 7 biosynthetic clusters, coding for unknown non-ribosomally synthesized peptides, and of tpc1/geo1 and tpc3/geo2 showing substantial similarity to terpene cyclase-encoding clusters, which in other microorganisms are known to produce geosmin, the sesquiterpene that provide the soil with its characteristic smell.

\section{Global and pathway-specific control of gene expression, and DNA repair mechanisms in S. Erythraea px}

Antibiotic production is under global and local pathwayspecific control. At the same time, there is evidence that genetic manipulation of RNA polymerase and/or ribosome may influence the control of secondary metabolism $[31,32]$. Mutations affecting the basic transcription and translation machineries were thus expected in the 
erythromycin- overproducing strain (Table 4). However, unexpectedly, several inactivating mutations mapped in a number of genes, whose products were thought to play fundamental roles in these processes including nusB (SACE_2076) coding for NusB antiterminator factor and efp (SACE_2075) encoding the elongation factor $\mathrm{P}$ (EF-P). Both these genes, which are organized in a putative conserved operon also including pep $Q$ gene coding for Xaa-Pro dipeptidase, were inactivated by frameshift mutations (Figure 8A).

NusB participates together with NusE/S10 protein in processive transcription antitermination. NusB and NusE, bind to form a heterodimer, which interacts with a specific boxA site on the RNA. The NusB/NusE/boxA RNA ternary complex interacts with the RNA polymerase transcription complex, stabilizing it and allowing transcription past premature termination points [37-40]. NusB is essential to suppress transcription termination in the ribosomal RNA ( $r r n$ ) operons. Compared to wild type Escherichia coli, about two-fold decreased RNA polymerase density was observed by electron microscopy over $23 \mathrm{~S}$ genes in a $n u s B$ mutant [41]. As a consequence, the fraction of total RNA polymerase engaged in transcribing the $r r n$ operons is significantly reduced in a $n u s B$ mutant. Similar reduction is observed during the stringent response, when (p)ppGpp binds RNA polymerase and changes the global transcriptional profile, decreasing the synthesis of translational machinery and increasing the transcription of biosynthetic genes including those coding for antibiotics in actinomycetes [32,42-48].

EF-P is a highly conserved protein that is essential for protein synthesis in several bacteria including $E$. coli [49]. It has been suggested that EF-P plays a role in translational fidelity, prevents entry of fMet-tRNA into the A-site enabling it to bind to the $50 \mathrm{~S} \mathrm{P}$-site, and promotes a ribosome-dependent accommodation of fMettRNA into the 70S P-site [50,51]. In Bacillus subtilis genetic inactivation of EF-P abolished spore formation without affecting growth [52]. Although it is difficult to predict the effects of EF-P inactivation on erythromycin production, it is relevant to note that erythromycin production in S. erythraea is stimulated by streptomycinresistance mutations [31] and that EF-P was shown to protect $16 \mathrm{~S}$ rRNA near the G526 streptomycin and the S12 and mRNA binding sites (30S T-site) [50].

In addition to EF-P, other genetic changes occurs in the Px strains affecting the translational machinery, including missense mutations affecting SACE_5926 (infB) coding for the translation initiation factor 2 , (SACE_0799) (metS) coding form methionyl-tRNA synthetase, SACE_0443 (cysS paralog) encoding a cysteinyl-tRNA synthatase, SACE_3403 (gatA) coding for A subunit of Asp-tRNA Asn/Glu-tRNA Gln amidotransferase, and a frameshift mutation inactivating a paralogous gene (SACE_5919) coding for pseudouridine synthase. These findings emphasize the importance of the translational machinery as a potential target for improvement of antibiotic-producing strains.

The mutations affecting the basic transcription and translation machineries may in turn affect pathway-specific control accounting for the global changes in transcriptional profile, which was observed in Px strain with respect to NRRL 2338 (Figures 4 and 5). As previously mentioned, a total of 459 and 220 genes were up-regulated during growth phase a in Px and in NRRL 2338 respectively. Among these DEGs, 138 were up-regulated in both strains, while 335 and 114 were specific for Px

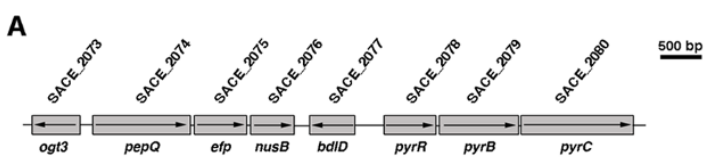

B

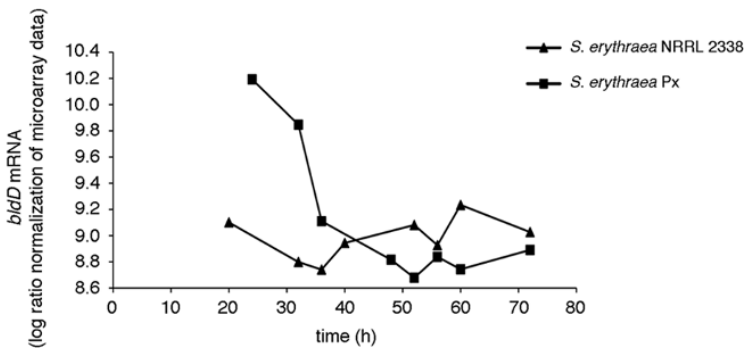

Figure 8 Expression of bldD during growth of S. erythraea NRRL 2338 and S. erythraea Px. A) Genetic map of the chromosomal region spanning the pepQ-efp-nusB and bldD genes. B) bldD mRNA levels are expressed as average of log ratio normalized microarray duplicate data (RMA). bldD expression values from S. erythraea NRRL 2338 and S. erythraea Px do not vary more than 1.58\% and $2.61 \%$, respectively, on the average. 
and NRRL 2338, respectively (Additional file 3, 4, 5). Within the list of genes specifically up-regulated in the Px strain, we found most of the genes belonging to the ery biosynthetic gene cluster. Regulation of the ery cluster was found to be altered also in another classically improved S. erythraea strain, which exhibited prolonged expression of antibiotic biosynthetic genes compared to the wild type during fermentation [34].

In addition to the ery cluster, SACE_1456 (mmsA2) coding for methylmalonate semialdehyde dehydrogenase was also found to be up-regulated in Px. This enzyme provides 6DEB biosynthesis with propionyl-CoA and (2S)-methylmalonyl-CoA precursors (Figure 6) and was found to be up-regulated in a rifampicin-resistant erythromycin-overproducing mutant of S. erythraea [32]. Moreover, the expression of bldD (SACE_2077), coding for a key developmental regulator that seems to regulate the ery cluster positively, was also up- regulated during phase a in the Px strain (Figure 8B). Intriguingly, this gene maps immediately downstream of the pepQ-efp$n u s B$ locus in the genome of S. erythraea (Figure 8A). In addition to the ery cluster and associated CDSs, the genes belonging to the $p k s 6$ and tpc5 (geo3) biosynthetic clusters were also found to be up-regulated in this strain along with nusA (SACE_5927 coding for transcriptional termination/antitermination factor NusA), ribosomal protein operons with the associated genes infA (SACE_6808 coding for translation initiation factor IF1), tufA (SACE_6838 coding for elongation factor EF1A), fusA (SACE_6839 coding for translation elongation factor G), and F0F1 ATPase. In contrast, in the list of genes specifically up-regulated in the NRRL 2338, we found many genes related to the TCA cycle including fumB (SACE_1784 coding for class I fumarate hydratase), fumC (SACE_0930 coding for class I fumarate hydratase), korA (SACE_3927 coding for 2- oxaloglutarate ferredoxin oxidoreductase alpha subunit), the urea cycle (ureA) (SACE_0634 coding for urease gamma subunit) or the PRPP biosynthesis (prsA) (SACE_0816).

In addition to changes in basic transcription and translation machineries, mutations in genes coding for transcriptional factors and signal transduction proteins may contribute to alter pathway-specific control. In particular, 13 genes coding for transcriptional factors and 5 genes encoding two-component system proteins were affected by missense or frameshift mutations in Px (Table 4). However, in the absence of functional data, the effects of such mutations on secondary metabolism may be only hypothesized on the basis of proximity of the mutated regulatory genes to the biosynthetic cluster for secondary metabolites. For instance, SACE_3079, coding for a LysR family transcriptional regulator and affected by a frame shift mutation in the same strain, is genetically linked to a hydrogenase operon (also containing gmhA genes, which were shown to be inactivated by frameshift mutations in $\mathrm{Px}$ ) and may be involved in its regulation. Similarly, SACE_4500, encoding a GntR family transcriptional regulator that is inactivated by a frameshift mutation in Px, maps close to sarcosine oxidase operon. Sarcosine oxidase links glycerophospholipid metabolism to erythromycin biosynthesis catalyzing the oxidative demethylation of sarcosine to glycine, whose metabolism provides the biosynthesis of 6DEB with precursors (Figure 6).

Px strain was obtained by multiple cycle of mutagenesis by chemical ( $N$-methyl- $N$-nitro- $N$ - nitrosoguanidine) or radiation (X-ray and UV) agents. The sequence data suggests that the mutagenesis/selection process to erythromycin-overproduction phenotype might have been accelerated by selection of mutator phenotype. This hypothesis is supported by missense and frameshift mutations affecting genes involved in DNA replication and repair. In particular, SACE_0826 ( $m f d$ ) coding for transcription/repair coupling factor, SACE_4427 (mug) coding for G/U mismatch-specific DNA glycosidase and SACE_5437 (polA) were inactivated by frameshift mutations, while missense mutations affected other genes involved in these processes (Table 5).

\section{Conclusions}

Overall our findings demonstrate that the phenotypes of the erythromycin-overproducing strain S. erythraea Px are associated with a large number of genetic changes with respect to the reference strain NRRL 2338. Mutations affect 227 CDSs, corresponding to about 3\% of CDSs of S. erythraea genome. Although certain mutations may be neutral in term of improved antibiotic production, and may have been favored by the mutator background of Px, a considerable number of them map within genes coding for key enzymes involved in central carbon and nitrogen metabolism, and biosynthesis of secondary metabolites, redirecting common precursors toward erythromycin biosynthesis. Several mutations inactivate genes coding for proteins that play fundamental roles in basic transcription and translation machineries including the transcription anti-termination factor NusB and the transcription elongation factor Efp, and genes coding for pleiotropic or pathway-specific regulators, with dramatic effects on global expression profile. The comparison of Px and NRRL 2338 at both genomic and transcriptomic levels not only contributed to elucidate the molecular mechanism underlying the overproduction of erythromycin, but also revealed new possible targets suitable for rationale improvement of industrial antibiotic-producing strains. However, as most of mutated genes are not directly related to the erythomycin over-production, next effort will be to test the genome-assisted predictions by experimental verification. 


\section{Methods}

\section{Bacterial strains and media}

S. erythraea wild type strain NRRL2338 was a gift of S. Donadio (KtedoGen, Milan, Italy). This strain has been deposited at the American Type Culture Collection. S. erythraea $\mathrm{Px}$ is an erythromycin-overproducing strain that was obtained by the traditional mutate-and-screen method over a period of about 10 years. The strains were stored in 1 - $\mathrm{ml}$ cryotubes at $-80^{\circ} \mathrm{C}$ as frozen mycelium in YS medium containing $15 \%$ glycerol at a biomass concentration of approximately $0.25 \mathrm{~g}$ dry cell weight $(\mathrm{DCW}) \mathrm{ml}^{-1}$, or at $-20^{\circ} \mathrm{C}$ as spores in $20 \%$ glycerol (in distilled water) at a title of approximately $5 \times 10^{8} \mathrm{ml}^{-1}$. The composition (per liter) of the complete media used in this study is reported in Table 8 . When requested all media were agarized at a concentration of $1.8 \%$. The composition (per liter) of the nutrient broth agar in the microbiological assays with Micrococcus luteus tester strain was: $3 \mathrm{~g}$ beef extract, $5 \mathrm{~g}$ tryptone, $15 \mathrm{~g} \mathrm{NaCl}, 15 \mathrm{~g}$ agar.

\section{Preparation of spores}

Concentrated spore suspensions $\left(5 \times 10^{8} \mathrm{ml}^{-1}\right)$ are crucial for purposes like starting reproducible cultures for physiological or fermentation studies. To prepare spores adapted to the conditions of liquid medium, spores were spread on the same medium with agar. Mycelium with spores was strongly attached to the surface agar, thus making impossible to collect spores without agar traces. Therefore, strains have been grown on cellophane discs, as described in [53]. The cellophane discs were sterilized in distilled water and then placed on agar, and the inoculum was spread on cellophane using a glass stick. After two weeks, spores (control in microscope) were easily scraped from cellophane and stored in $20 \%$ glycerol at $-20^{\circ} \mathrm{C}$.

\section{Growth conditions}

For shake-flask experiments, spores in frozen aliquots were collected by centrifugation, re- suspended in medium 707 (for rehydration), and readily separated by vortexing. Individual aliquots (about $5 \times 10^{8}$ spores) were used to inoculate each $500 \mathrm{ml}$ baffled Erlenmeyer flask containing $50 \mathrm{ml}$ of the liquid media described above. Cultures were incubated at $30^{\circ} \mathrm{C}$ with shaking at $250 \mathrm{rpm}$. Bioreactor cultures were carried out on Minifors mini-fermenters (Infors AG, Bottmingen, $\mathrm{CH}$ ) that operated with a working volume of $1.5 \mathrm{l}$. Stirring was provided by Rushton-type impellors rotating at $250 \mathrm{rpm}$. Sterile air was supplied through a sparger. The bioreactors were equipped with $\mathrm{pH}$ electrode, $\mathrm{pO} 2$ electrode (polarographic), antifoam probe and $\mathrm{Pt}-100$.

\section{Erythromycin assays}

Erythromycin production in solid media was assayed by bioassay. To this purpose, S. erythraea strains were grown in solid media $(30 \mathrm{ml})$ in Petri dishes $(8.5 \mathrm{~cm})$. After desired time of cultivation, agar discs of $1.6 \mathrm{~cm}$ in diameter (with mycelium on the surface) were removed and placed into empty Petri dishes (diameter $8.5 \mathrm{~cm}$ ). Petri dishes were then filled with soft nutrient agar seeded with Micrococcus luteus. Diameters of the zone of inhibition were measured after 2 days of incubation at $37^{\circ} \mathrm{C}$. Agar discs containing defined amounts of $>$ 95\% pure erythromycin A (Sigma) were used as a reference. In liquid media, erythromycin was extracted as described [54]. Five hundred $\mu \mathrm{L}$ of $n$-butylacetate were added to $500 \mu \mathrm{L}$ of broth fermentation, samples were vortexed for $5 \mathrm{~min}$ and centrifuged at $9500 \mathrm{~g}$ for 15 min. Then the organic phase was mixed with $500 \mu \mathrm{L}$ buffer $25 \mathrm{mM} \mathrm{K}_{2} \mathrm{PO}_{4} \mathrm{pH} 5$ and centrifuged at $800 \mathrm{~g}$ for $5 \mathrm{~min}$. Ten $\mu \mathrm{L}$ of the aqueous phase was injected into HPLC.

HPLC analyses were carried out using an Agilent 1100 Series HPLC system equipped with security guard Cartridges (C18 ODS $4 \times 3 \mathrm{~mm}$ ) and a Phenomenex-luna 5 $\mu \mathrm{C} 18$ (2) $100 \AA$ column $(250 \times 4.6 \mathrm{~mm})$. An isocratic elution mode was used according to [55] Tsuji and Goetz

Table 8 Composition of the media used in this study

\begin{tabular}{|c|c|c|}
\hline Medium & Composition (per liter) & $\mathrm{pH}$ \\
\hline \multicolumn{3}{|c|}{ Complex } \\
\hline Seed medium (SM) & $4 \mathrm{~g}$ peptone, $4 \mathrm{~g}$ yeast extract, $2 \mathrm{~g} \mathrm{KH}_{2} \mathrm{PO}_{4}, 4 \mathrm{~g} \mathrm{~K}_{2} \mathrm{HPO}_{4}, 0.5 \mathrm{~g} \mathrm{MgSO}_{4} 7 \mathrm{H}_{2} \mathrm{O}, 10 \mathrm{~g}$ glucose & 7.2 \\
\hline Yeast starch (YS) & $2 \mathrm{~g}$ yeast extract, $10 \mathrm{~g}$ soluble starch & 7.3 \\
\hline Oat meal yeast (OMY) & $40 \mathrm{~g}$ oatmeal, $1 \mathrm{~g}$ yeast extract & $\begin{array}{l}6.8- \\
7.0\end{array}$ \\
\hline $\begin{array}{l}\text { Soluble complete medium } \\
\text { (SCM) }\end{array}$ & $20 \mathrm{~g}$ soytone, $15 \mathrm{~g}$ soluble starch, $10.5 \mathrm{~g}$ morpholinepropanesulfonic acid, $1.5 \mathrm{~g}$ yeast extract, $0.1 \mathrm{~g} \mathrm{CaCl} 2$ & 7.2 \\
\hline \multicolumn{3}{|c|}{ Chemically defined } \\
\hline MM-101 & $\begin{array}{c}7 \mathrm{~g} \mathrm{NH}_{4} \mathrm{Cl}, 3 \mathrm{~g} \mathrm{KH}_{2} \mathrm{PO}_{4}, 7 \mathrm{~g} \mathrm{~K}_{2} \mathrm{HPO}_{4}, 0.25 \mathrm{~g} \mathrm{MgSO}_{4} \cdot 7 \mathrm{H}_{2} \mathrm{O}, 0.0138 \mathrm{~g} \mathrm{CaCl}_{2} \cdot 2 \mathrm{H}_{2} \mathrm{O}, 10 \mathrm{~g} \text { glucose, } 2 \mathrm{ml} \text { trace } \\
\text { solution element (TSE)a }\end{array}$ & 6.9 \\
\hline
\end{tabular}

aWhen indicated $1.0 \mathrm{~g}$ casamino acids (Difco, Detroit, Mich.) and/or $2 \mathrm{ml}$ (per liter) trace solution element (TSE) were added. The TSE solution composition (per liter) was: $40 \mathrm{mg} \mathrm{ZnCl}, 200 \mathrm{mg} \mathrm{FeCl} \cdot 6 \mathrm{H}_{2} \mathrm{O}, 10 \mathrm{mg} \mathrm{CuCl} 2 \cdot 2 \mathrm{H}_{2} \mathrm{O}, 10 \mathrm{mg} \mathrm{MnCl} \cdot 4 \mathrm{H}_{2} \mathrm{O}, 10 \mathrm{mg} \mathrm{Na}_{2} \mathrm{~B}_{4} \mathrm{O}_{7} \cdot 10 \mathrm{H}_{2} \mathrm{O}, 10 \mathrm{mg}\left(\mathrm{NH}_{4}\right)_{6} \mathrm{Mo}_{7} \mathrm{O}_{24} \cdot 4 \mathrm{H}_{2} \mathrm{O}$ 
(1978). Mobile phase was as follows: acetonitrile-methanol-0.2 $\mathrm{M}$ ammonium acetate-water (45:10:10:35) at $\mathrm{pH}$ 7.8 , at flow rate of $1 \mathrm{ml} \mathrm{min}^{-1}$ at $25^{\circ} \mathrm{C}$ using $\mathrm{UV}$ detector at $215 \mathrm{~nm}$. Quantification of the erythromycin was achieved using calibration curves of peak area against injected concentration of the various erythromycin standards A, B, C (European Pharmacopoeia HPLC assay).

\section{DNA procedures}

High molecular weight genomic DNA was extracted from $S$. erythraea strains grown in $50 \mathrm{ml}$ of SM medium with shaking at $28^{\circ} \mathrm{C}$ for 5 days $(120 \mathrm{~h})$. After centrifugation, the mycelium was re-suspended in $10 \mathrm{ml} \mathrm{SET}$ buffer (75 mM NaCl, $25 \mathrm{mM}$ EDTA, $20 \mathrm{mM}$ Tris- Cl $\mathrm{pH} 7.5$ ) and incubated in the presence of $5 \mathrm{mg} \mathrm{ml}^{-1}$ lysozyme for $30^{\prime}$ at $37^{\circ} \mathrm{C}$. Samples were sonicated (Sonifer sonicator Model 250/240, Brain Ultrasonic Corporation) 3 times for $30 \mathrm{sec}$, and incubated in the presence of $20 \mathrm{mg} \mathrm{ml}-1$ Proteinase $\mathrm{K}$ and $1.2 \%$ sodium dodecyl sulfate (SDS) for $2 \mathrm{~h}$ at $55^{\circ} \mathrm{C}$. Nucleic acids were extracted by fenol-chloroform:isoamylic alcohol (24:1) according to standard procedure (Sambrook and Russell, 2001) and RNA removed using $15 \mu \mathrm{g}$ ml-1 ribonuclease A. After fenol-chloroform:isoamylic alcohol (24:1) extraction and ethanol-precipitation, high molecularweight DNA was collected by spooling using Shepherd's crooks [56,57].

Sequencing and assembly of the S. Erythraea px genome Whole-genome shotgun DNA sequencing of S. erythraea Px genome was performed by MWG Biotech (Eurofins MWG Operon, Ebersberg, Germany) using frequently cutting restriction enzymes and 2- to $10-\mathrm{kbp}$ fragments cloned into plasmid vectors. Cosmids (32-46 kbp inserts) were also generated from genomic DNA and end-sequenced to provide additional read-pair information, increase coverage of selected regions, and fill the gaps. Remaining gaps and ambiguities were closed using PCR products from specifically designed oligonucleotide primers. Sequence assembly was done using the Phrap assembler43 and editing was done using consed version 14 . Repeats were resolved by doing a miniassembly for the individual sections of the genome and the resulting consensus was integrated into the main genome assembly.

\section{RNA extraction and RNA microarray experiments}

The S. erythraea Custom GeneChip used for the NRRL2338 strain gene expression profiling by Peano et al. [24] was used here to analyze the time course gene expression profiling of the Px strain. The sequences of all probes present on the GeneChip were compared with the genomic sequence of the Px strain using
BLASTN [58], finding a perfect match for all of them, both for similarity (100\%) and length (25 bp).

For each time point, RNA was extracted from mycelium pellets deriving from 1-ml culture samples using the GeneElute ${ }^{\mathrm{TM}}$ total RNA Purification Kit (SIGMA), recovering it in $50 \mu \mathrm{l}$ of Elution Solution. After extraction RNAs were quantified with a NanoDrop spectrophotometer (NanoDrop Technologies) and analyzed by capillary electrophoresis on a Agilent Bioanalyzer (Agilent). The RNA samples showing an RIN (RNA Integrity Number, a quality parameter calculated by the instrument software) value higher than 7 were processed for microarray hybridization, following the instructions for "Prokaryotic Target Preparation" (Affymetrix GeneChip ${ }^{\circledR}$ Expression Analysis Technical Manual). The protocol consists in cDNA synthesis by reverse transcription (starting with $10 \mu \mathrm{g}$ RNA), followed by cDNA fragmentation with DNase I and labeling with Terminal Deoxynucleotidyl Transferase. The labeled cDNAs were then hybridized for $16 \mathrm{~h}$ at $50^{\circ} \mathrm{C}$ on individual GeneChips. After hybridization, GeneChips were washed and stained with streptavidin-conjugated phycoerythrin by using the Fluidic Station FS450 (Affymetrix) following the FS450_0005 Protocol. Fluorescent images of the microarrays were acquired using a GeneChip Scanner 3000 (Affymetrix). All raw data files are available in Gene Expression Omnibus under accession number GSE30600.

\section{Genomic comparison and variation detection}

The software Nucmer from the MUMmer package [59] (http://mummer.sourceforge.net) was used to investigate the presence of possibly large genomic rearrangement. All other comparative analyses have been carried out using the BLAST suite from NCBI (http://www.ncbi. nlm.nih.gov/BLAST,ftp://ftp.ncbi.nih.gov/blast), in particular the BLASTx and BLASTn programs, whose results were then parsed using home-made scripts. The circular plots were generated by an home-made Python script. The sequences of the 227 genes (SACX_) affected by mutations in the Px strain as compared to corresponding genes (SACE_) in the parental NRRL2338 strain have been submitted to GeneBank (accession numbers: from JN392509 to JN392716 in Additional file 7).

\section{Microarray data analysis}

The quality of the raw data obtained from microarray hybridization was assessed considering the MAS5.0 (Microarray Suite/Software, Affymetrix) control parameters after a global scaling at a target intensity of 100 . Quality and control parameters as well as box plots of raw intensities highlighted the overall high quality of the data set and the absence of any outlying sample. Probe 
level data was converted to expression values using both the Robust Multi- array Average (RMA) procedure [60] and the MAS5.0 algorithms. In the former case, PM values (Perfect Match) were background-adjusted, normalized using invariant set normalization, and log transformed. In the latter case, intensity levels were normalized using the Global Scaling option to target value (i.e. TGT = 100).

Genes characterized by a statistically significant modulation of the expression level during the growth time course (within-class temporal differential expression) were identified using the EDGE software package, which is based on the Optimal Discovery Procedure [61] and allows identifying genes that are differentially expressed between two or more different biological conditions or to perform significance analysis on time course experiments [62]. Whereas other methods employ statistics essentially designed for testing one gene at a time (e.g. t-statistics and F-statistics), the ODP uses all relevant information from all genes to test each gene for differential expression, thus improving the power of the test. In the particular case of a time course, ODP takes into account the ordering and spacing information provided by the time points.

Briefly, we have tested each gene by first fitting a model (e.g. natural cubic sp-lines) under the null hypothesis that there is no differential expression, and then under the alternative hypothesis that there is differential expression. A statistic is calculated to compare the goodness of fit of the two models under the two different hypotheses. The statistic is a quantification of evidence for transcriptional modulation, and the larger it is the more differentially expressed the gene appears to be. Once the statistic is calculated for each gene, a significance cut-off is applied using a false discovery rate criterion. This process, based on the calculation of the null distribution of the statistics when there is no differential expression, is accomplished through a data re-sampling technique and results in the q-value. Modulated genes are finally selected based on the q-value threshold and, eventually on a fold change limit. Differentially expressed genes were selected considering each time point replicate as an independent entry and setting the confidence threshold at q-value $\leq 0.001$. Hierarchical clustering and Eisen's maps were used to group modulated genes and samples in the software package dChip. Before clustering, the expression values for a gene across all samples were standardized (linearly scaled) to have mean 0 and standard deviation 1 , and these standardized values were used to calculated correlations between genes and samples and served as the basis for merging nodes. Hierarchical agglomerative clustering was carried out using Pearson correlation coefficient as distance metric and centroid as linkage method.
Chromosomal regions presenting a between-class temporal differential expression were identified using PREDA $[35,63,64]$. PREDA is a bioinformatic tool developed under $\mathrm{R}$ statistical environment for the identification of differentially expressed chromosomal regions, which accounts for variations in gene distance and density. PREDA consists of three main steps: i) computation of a statistic for ranking probes in order of strength of evidence for an expression feature; ii) adaptive bandwidth smoothing of the statistic after sorting the statistical scores according to the chromosomal position of the corresponding genes; and iii) application of a permutation test to identify differentially expressed chromosomal regions with a q-value correction for multiple tests. Transcriptional and structural information are locally integrated smoothing, along the chromosomal coordinate, an expression statistic. The smoothing procedure is approached as a non-parametric regression problem using a local variable bandwidth kernel estimator. A permutation scheme is used to identify differentially expressed regions under the assumption that each gene has a unique neighborhood and that the corresponding smoothed statistic is not comparable with any statistic smoothed in other regions of the genome. The permutation process over $\mathrm{B}$ random assignments allows defining a null smoothed statistic for any chromosomal position. The significance of the differentially expressed regions (i.e. the p-value) is computed as the probability that the random null statistic exceeds the observed statistic over $\mathrm{B}$ permutations. Once the distribution of empirical $\mathrm{p}-$ values has been generated, the q-value is used to identify differentially expressed chromosomal regions according to Storey and Tibshirani [65].

\section{Additional material}

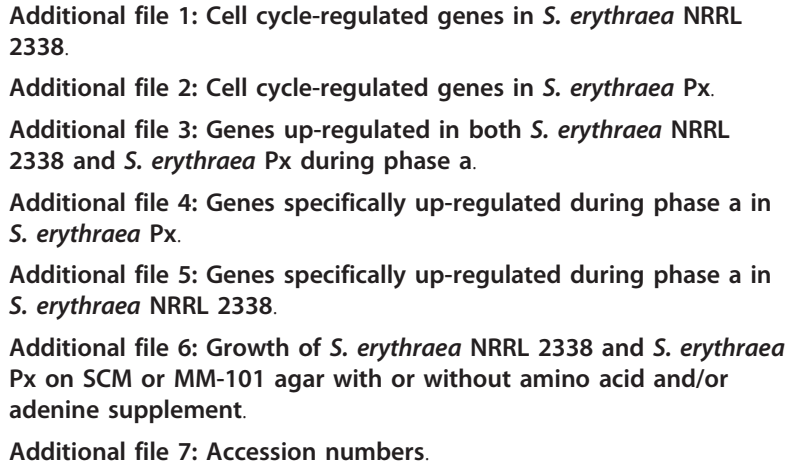

\section{Acknowledgements}

We are grateful to Prof. S. Donadio for gift of S. erythraea wild type strain NRRL2338, and Dr. S. M. Tredici and Dr. C. Marcuccio for technical assistance. This work was partially supported by grant from the Italian Ministry for University and Research (MIUR): MIUR Cofin 2008 - prot. 2008232P4H to PA, 
FIRB-MIUR NG-LAB Research Program, Project RBLA03ER38_004, to GDB, and from the Lombardia Region: "Accordo Quadro CNR- Regione Lombardia Sottoprogetto 4, Piattaforma 2" for Antibiotics development. The funders had no role in study design, data collection and analysis, decision to publish, or preparation of the manuscript.

\section{Author details}

${ }^{1}$ Institute of Biomedical Technologies, National Research Council, Via Fratelli Cervi 93, 20090 Segrate, Milano, Italy. ${ }^{2}$ Dipartimento di Scienze e Tecnologie Biologiche ed Ambientali, Università del Salento, Via Monteroni, 73100 Lecce, Italy. ${ }^{3}$ Operative Unit of Lecce, CNR - Institute of Sciences of Food Production (ISPA), via Provinciale Lecce-Monteroni, 73100 Lecce, Italy. ${ }^{4}$ Center for Genome Research, Dept. of Biomedical Sciences, University of Modena and Reggio Emilia, Via G. Campi 287, 41100 Modena, Italy.

\section{Authors' contributions}

Conceived and designed the experiments: GDB PA. Performed the experiments: CP AT GC DP SB. Analyzed the data: CP, SB, GDB, PA. Wrote the paper: $C P, P A$. All authors read and approved the final manuscript.

\section{Competing interests}

The authors declare that they have no competing interests.

Received: 8 September 2011 Accepted: 8 March 2012

Published: 8 March 2012

\section{References}

1. Champness WC, Chater KF: The regulation and integration of antibiotic production and morphological differentiation in Streptomyces spp Washington, DC: American Society for Microbiology; 1994.

2. Adrio JL, Demain AL: Genetic improvement of processes yielding microbial products. FEMS Microbiol Rev 2006, 30(2):187-214.

3. Baltz RH: Genetic methods and strategies for secondary metabolite yield improvement in actinomycetes. Antonie Van Leeuwenhoek 2001, 79(34):251-259.

4. Vinci VA, Byng G: In Manual of Industrial Microbiology and Biotechnology.. 2 edition. Edited by: Demain AL, Davies JE, Atlas RM, Cohen G, Hershberger CL, Hu W-S, Sherman DH, Willson RC, Wu JHD. Washington, DC: American Society for Microbiology; 1999:103-113.

5. Blanchard AP, Hood L: Sequence to array: probing the genome's secrets. Nat Biotechnol 1996, 14(13):1649.

6. Bro C, Nielsen J: Impact of 'ome' analyses on inverse metabolic engineering. Metab Eng 2004, 6(3):204-211.

7. Lander ES: The new genomics: global views of biology. Science 1996, 274(5287):536-539.

8. Lander ES: Array of hope. Nat Genet 1999, 21(1 Suppl):3-4

9. Lee SY, Lee DY, Kim TY: Systems biotechnology for strain improvement. Trends Biotechnol 2005, 23(7):349-358.

10. Stafford DE, Stephanopoulos G: Metabolic engineering as an integrating platform for strain development. Curr Opin Microbiol 2001, 4(3):336-340.

11. van der Werf MJ, Jellema RH, Hankemeier T: Microbial metabolomics: replacing trial-and-error by the unbiased selection and ranking of targets. J Ind Microbiol Biotechnol 2005, 32(6):234-252.

12. Donadio S, Monciardini P, Sosio M: Polyketide synthases and nonribosomal peptide synthetases: the emerging view from bacterial genomics. Nat Prod Rep 2007, 24(5):1073-1109.

13. Katz L, Donadio S: Macrolides. Biotechnology 1995, 28:385-420.

14. Leadlay PF: Combinatorial approaches to polyketide biosynthesis. Curr Opin Chem Biol 1997, 1(2):162-168.

15. Hutchinson CR, Decker H, Madduri K, Otten SL, Tang L: Genetic control of polyketide biosynthesis in the genus Streptomyces. Antonie Van Leeuwenhoek 1993, 64(2):165-176.

16. McDaniel R, Licari P, Khosla C: Process development and metabolic engineering for the overproduction of natural and unnatural polyketides. Adv Biochem Eng Biotechnol 2001, 73:31-52.

17. Corcoran JW: Biochemical mechanisms in the biosynthesis of the erythromycins. In Antibiotics IV Biosynthesis. Edited by: Corcoran JW. New York: Springer-Verlag edn; 1981:132-174.
18. Cortes J, Haydock SF, Roberts GA, Bevitt DJ, Leadlay PF: An unusually large multifunctional polypeptide in the erythromycin-producing polyketide synthase of Saccharopolyspora erythraea. Nature 1990, 348(6297):176-178

19. Donadio S, Staver MJ, MCAlpine JB, Swanson SJ, Katz L: Modular organization of genes required for complex polyketide biosynthesis. Science 1991, 252(5006):675-679.

20. Donadio S, Staver MJ: IS1136, an insertion element in the erythromycin gene cluster of Saccharopolyspora erythraea. Gene 1993, 126(1):147-151.

21. Weber JM, Leung JO, Maine GT, Potenz RH, Paulus TJ, DeWitt JP: Organization of a cluster of erythromycin genes in Saccharopolyspora erythraea. J Bacteriol 1990, 172(5):2372-2383.

22. Reeves AR, English RS, Lampel JS, Post DA, Vanden Boom TJ: Transcriptional organization of the erythromycin biosynthetic gene cluster of Saccharopolyspora erythraea. J Bacteriol 1999, 181(22):7098-7106.

23. Oliynyk M, Samborskyy M, Lester JB, Mironenko T, Scott N, Dickens S, Haydock SF, Leadlay PF: Complete genome sequence of the erythromycin-producing bacterium Saccharopolyspora erythraea NRRL23338. Nat Biotechnol 2007, 25(4):447-453.

24. Peano C, Bicciato S, Corti G, Ferrari F, Rizzi E, Bonnal RJ, Bordoni R, Albertini A, Bernardi LR, Donadio S, et al: Complete gene expression profiling of Saccharopolyspora erythraea using GeneChip DNA microarrays. Microb Cell Fact 2007, 6:37.

25. Chang X, Liu S, Yu YT, Li YX, Li YY: Identifying modules of coexpressed transcript units and their organization of Saccharopolyspora erythraea from time series gene expression profiles. PLoS One 8 5:e12126.

26. Elliot M, Damji F, Passantino R, Chater K, Leskiw B: The bldD gene of Streptomyces coelicolor A3(2): a regulatory gene involved in morphogenesis and antibiotic production. J Bacteriol 1998, 180(6):1549-1555.

27. Elliot MA, Bibb MJ, Buttner MJ, Leskiw BK: BldD is a direct regulator of key developmental genes in Streptomyces coelicolor A3(2). Mol Microbiol 2001, 40(1):257-269.

28. Chng C, Lum AM, Vroom JA, Kao CM: A key developmental regulator controls the synthesis of the antibiotic erythromycin in Saccharopolyspora erythraea. Proc Natl Acad Sci USA 2008, 105(32):11346-11351.

29. Reeves AR, Cernota WH, Brikun IA, Wesley RK, Weber JM: Engineering precursor flow for increased erythromycin production in Aeromicrobium erythreum. Metab Eng 2004, 6(4):300-312.

30. Reeves AR, Brikun IA, Cernota WH, Leach Bl, Gonzalez MC, Weber JM: Effects of methylmalonyl-CoA mutase gene knockouts on erythromycin production in carbohydrate-based and oil-based fermentations of Saccharopolyspora erythraea. J Ind Microbiol Biotechnol 2006, 33(7):600-609.

31. Tanaka Y, Komatsu M, Okamoto S, Tokuyama S, Kaji A, Ikeda H, Ochi K: Antibiotic overproduction by rpsL and rsmG mutants of various actinomycetes. Appl Environ Microbiol 2009, 75(14):4919-4922.

32. Carata E, Peano C, Tredici SM, Ferrari F, Tala A, Corti G, Bicciato S, De Bellis $G$, Alifano P: Phenotypes and gene expression profiles of Saccharopolyspora erythraea rifampicin-resistant (rif) mutants affected in erythromycin production. Microb Cell Fact 2009, 8:18.

33. Zhang H, Wang Y, Wu J, Skalina K, Pfeifer BA: Complete biosynthesis of erythromycin A and designed analogs using $\mathrm{E}$. coli as a heterologous host. Chem Biol 2010, 17(11):1232-1240.

34. Lum AM, Huang J, Hutchinson CR, Kao CM: Reverse engineering of industrial pharmaceutical-producing actinomycete strains using DNA microarrays. Metab Eng 2004, 6(3):186-196.

35. Callegaro A, Basso D, Bicciato S: A locally adaptive statistical procedure (LAP) to identify differentially expressed chromosomal regions. Bioinformatics 2006, 22(21):2658-2666.

36. Katz L, Khosla C: Antibiotic production from the ground up. Nat Biotechnol 2007, 25(4):428-429.

37. Gopal B, Haire LF, Cox RA, Jo Colston M, Major S, Brannigan JA, Smerdon SJ, Dodson G: The crystal structure of NusB from Mycobacterium tuberculosis. Nat Struct Biol 2000, 7(6):475-478

38. Doherty GP, Meredith DH, Lewis PJ: Subcellular partitioning of transcription factors in Bacillus subtilis. J Bacteriol 2006, 188(11):4101-4110 
39. Luo X, Hsiao HH, Bubunenko M, Weber G, Court DL, Gottesman ME, Urlaub H, Wahl MC: Structural and functional analysis of the E. coli NusBS10 transcription antitermination complex. Mol Cell 2008, 32(6):791-802.

40. Weisberg RA: Transcription by moonlight: structural basis of an extraribosomal activity of ribosomal protein S10. Mol Cell 2008, 32(6):747-748.

41. Quan S, Zhang N, French S, Squires CL: Transcriptional polarity in rRNA operons of Escherichia coli nusA and nusB mutant strains. J Bacteriol 2005, 187(5):1632-1638.

42. Chakraburtty R, Bibb M: The ppGpp synthetase gene (relA) of Streptomyces coelicolor A3(2) plays a conditional role in antibiotic production and morphological differentiation. J Bacterio/ 1997, 179(18):5854-5861.

43. Martinez-Costa OH, Arias P, Romero NM, Parro V, Mellado RP, Malpartida F: A relA/spoT homologous gene from Streptomyces coelicolor $\mathrm{A} 3(2)$ controls antibiotic biosynthetic genes. J Biol Chem 1996, 271(18):10627-10634.

44. Ochi K: Metabolic initiation of differentiation and secondary metabolism by Streptomyces griseus: significance of the stringent response (ppGpp) and GTP content in relation to A factor. J Bacterio/ 1987, 169(8):3608-3616.

45. Ochi K: From microbial differentiation to ribosome engineering. Biosci Biotechnol Biochem 2007, 71(6):1373-1386.

46. Strauch E, Takano E, Baylis HA, Bibb MJ: The stringent response in Streptomyces coelicolor A3(2). Mol Microbiol 1991, 5(2):289-298.

47. Tala A, Wang G, Zemanova M, Okamoto S, Ochi K, Alifano P: Activation of dormant bacterial genes by Nonomuraea sp. strain ATCC 39727 mutanttype RNA polymerase. J Bacteriol 2009, 191(3):805-814.

48. Siculella L, Damiano F, di Summa R, Tredici SM, Alduina R, Gnoni GV, Alifano P: Guanosine $5^{\prime}$-diphosphate $3^{\prime}$-diphosphate (ppGpp) as a negative modulator of polynucleotide phosphorylase activity in a 'rare' actinomycete. Mol Microbiol 2010, 77(3):716-729.

49. Aoki H, Dekany K, Adams SL, Ganoza MC: The gene encoding the elongation factor $\mathrm{P}$ protein is essential for viability and is required for protein synthesis. J Biol Chem 1997, 272(51):32254-32259.

50. Aoki H, Xu J, Emili A, Chosay JG, Golshani A, Ganoza MC: Interactions of elongation factor EF-P with the Escherichia coli ribosome. FEBS J 2008, 275(4):671-681.

51. Blaha G, Stanley RE, Steitz TA: Formation of the first peptide bond: the structure of EF-P bound to the 70S ribosome. Science 2009, 325(5943):966-970

52. Ohashi $Y$, Inaoka T, Kasai K, Ito Y, Okamoto S, Satsu H, Tozawa Y, Kawamura F, Ochi K: Expression profiling of translation-associated genes in sporulating Bacillus subtilis and consequence of sporulation by gene inactivation. Biosci Biotechnol Biochem 2003, 67(10):2245-2253.

53. Kieser T, Bibb MJ, Buttner MJ, Chater KF, Hopwood DA: Practical Streptomyces genetics Norwich, England: The John Innes Foundation; 2000, Crowes.

54. Kamareia F, Attara H, Nikjahb S, Goodarzi M: Determination of erythromycins in fermentation broth using liquid phase extraction with back extraction combined with high performance liquid chromatography. Arabian Journal of Chemisty 2010, doi:10.1016/j. arabjc.2010.10.033.

55. Tsuji K, Goetz JF: HPLC as a rapid means of monitoring erythromycin and tetracycline fermentation processes. J Antibiot 1978, 31(4):302-308, Tokyo.

56. Sambrook J, Russell DW: Molecular Cloning. A Laboratory Manual Cold Spring Harbor, New York: Cold Spring Harbor Laboratory Press; 2001.

57. Vigliotta G, Tredici SM, Damiano F, Montinaro MR, Pulimeno R, di Summa R, Massardo DR, Gnoni GV, Alifano P: Natural merodiploidy involving duplicated rpoB alleles affects secondary metabolism in a producer actinomycete. Mol Microbiol 2005, 55(2):396-412.

58. Altschul SF, Gish W, Miller W, Myers EW, Lipman DJ: Basic local alignment search tool. J Mol Biol 1990, 215(3):403-410.

59. MUMMER. [http://mummer.sourceforge.net]

60. Irizarry RA, Hobbs B, Collin F, Beazer-Barclay YD, Antonellis KJ, Scherf U, Speed TP: Exploration, normalization, and summaries of high density oligonucleotide array probe level data. Biostatistics 2003, 4(2):249-264.

61. Storey JD, Dai JY, Leek JT: The optimal discovery procedure for largescale significance testing, with applications to comparative microarray experiments. Biostatistics 2007, 8(2):414-432.
62. Storey JD, Xiao W, Leek JT, Tompkins RG, Davis RW: Significance analysis of time course microarray experiments. Proc Natl Acad Sci USA 2005, 102(36):12837-12842

63. Ferrari F, Solari A, Battaglia C, Bicciato S: PREDA: an R-package to identify regional variations in genomic data. Bioinformatics 2011, 27(17):2446-2447.

64. Storey JD, Tibshirani R: Statistical significance for genomewide studies. Proc Natl Acad Sci USA 2003, 100(16):9440-9445.

65. Li C, Wong WH: Model-based analysis of oligonucleotide arrays: expression index computation and outlier detection. Proc Natl Acad Sci USA 2001, 98(1):31-36.

doi:10.1186/1475-2859-11-32

Cite this article as: Peano et al:: Comparative genomics and transcriptional profiles of Saccharopolyspora erythraea NRRL 2338 and a classically improved erythromycin over-producing strain. Microbial Cell Factories 2012 11:32.

\section{Submit your next manuscript to BioMed Central and take full advantage of:}

- Convenient online submission

- Thorough peer review

- No space constraints or color figure charges

- Immediate publication on acceptance

- Inclusion in PubMed, CAS, Scopus and Google Scholar

- Research which is freely available for redistribution

Submit your manuscript at www.biomedcentral.com/submit
Biomed Central 